\title{
Hypercubes clustering: a machine learning method for efficiently finding common sub-trajectories in spatiotemporal space and constructing trajectories models for prediction
}

This paper was downloaded from TechRxiv (https://www.techrxiv.org).

\section{LICENSE}

CC BY 4.0

SUBMISSION DATE / POSTED DATE

27-10-2021 / 01-02-2022

\section{CITATION}

Hsu, Oscar LiJen (2022): Hypercubes clustering: a machine learning method for efficiently finding common sub-trajectories in spatiotemporal space and constructing trajectories models for prediction. TechRxiv. Preprint. https://doi.org/10.36227/techrxiv.16884844.v1

$\mathrm{DOI}$ 
Hypercubes clustering: a machine learning method for efficiently finding common sub-trajectories in spatiotemporal space and constructing trajectories models for prediction

AUTHER : Oscar LiJen Hsu 



\section{ABSTRACT}

Common sub-trajectory clustering is to find similar trajectory segments. Existing clustering methods tend to overlook many of the relevant sub-trajectories; others require a road network as input; all are significantly slowed down considerably by large datasets. This study proposes a novel machine learning approach, called Hypercubes clustering. Hypercubes clustering transforms trajectories into a set of Hypercubes. This study further applies Hypercubes clustering to solving the Estimated Time of Arrival (ETA) problem to show a practical use. ETA, which is used to predict the travel time of a given GPS trajectory, has been extensively used in route planning. Deep learning has been widely applied to ETA prediction. However, prediction tasks involve some challenges, such as small data size, low precision of GPUs, high training loss, and low accuracy. In the training phase, a trajectory model is established using historical trajectories. In the prediction phase, ETA is calculated according to the model. The software of this study for ETA prediction is named HyperETA. The performance of Hypercubes clustering was compared with that of grid clustering (i.e., constant time technique) in terms of memory usage, computational speed and compared with a state-of-art method, TraClus, by assessing their accuracy. The results of HyperETA are compared with a deep-learningbased ETA method, called DeppTTE. The experiment results show that Hypercube clustering can identify common sub-trajectories more swiftly and with less memory usage than grid clustering. The accuracy of Hypercube clustering and HyperETA is superior to TraClus and DeppTTE, respectively. A few problems associated with deep learning are discussed in this study. 


\section{KEYWORDS}

Urban computing; similar trajectories; ridesharing paths; common sub-trajectories clustering; Estimated Time of Arrival; ETA; Deep Learning. 


\section{CONTENTS}

摘要 錯誤! 尚未定義書籤。

ABSTRACT .

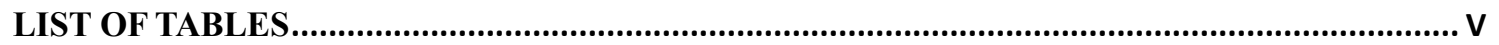

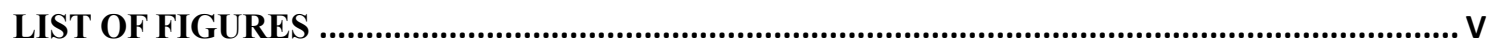

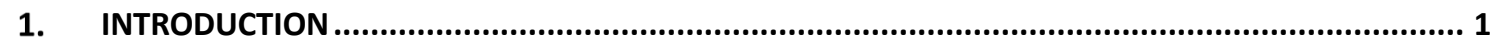

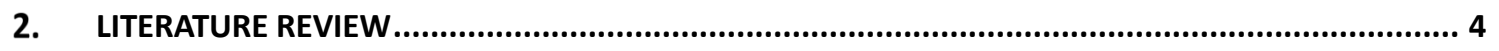

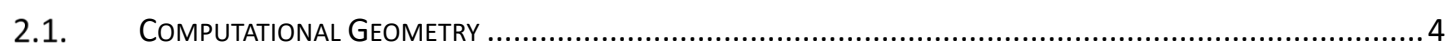

2.2. Methods of FInding Common Trajectory .......................................................................... 4

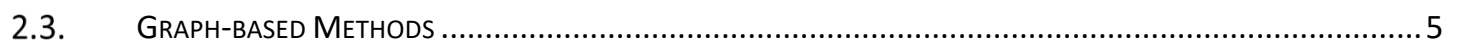

2.4. RIDESHARING SYSTEM AND GRID CLUSTERING .......................................................................

2.5. DeEP LeARning Methods ........................................................................................

3. DEFINITIONS OF COMMON SUB-TRAJECTORIES .................................................................. 9

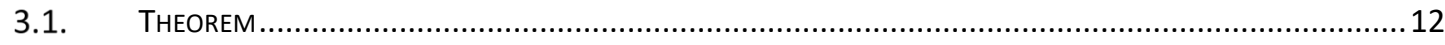

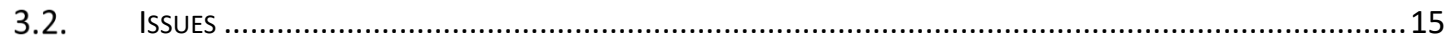

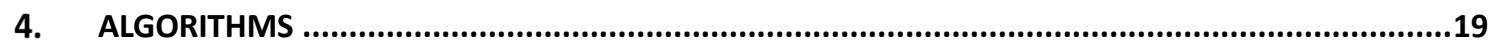

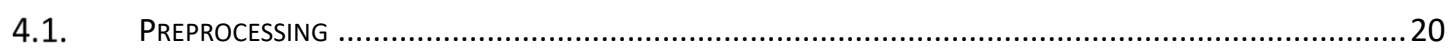

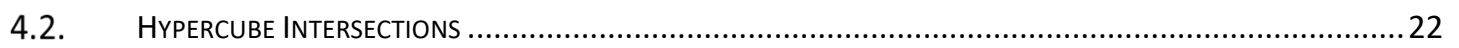

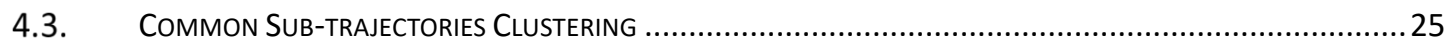

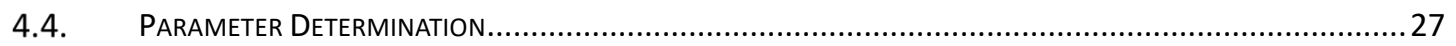

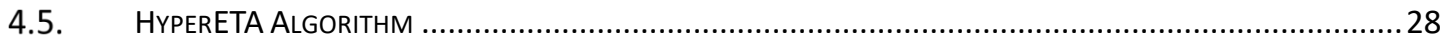

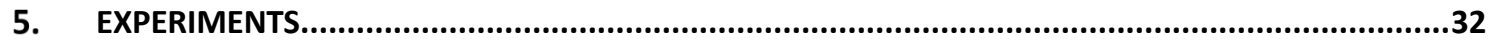

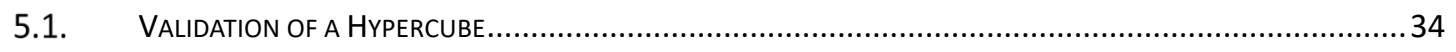

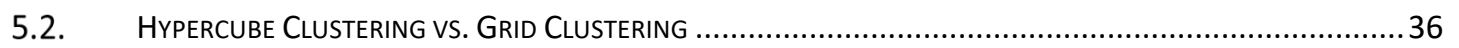

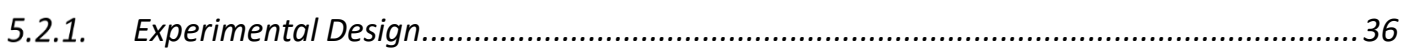

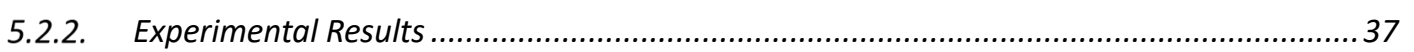

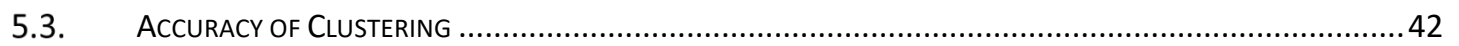

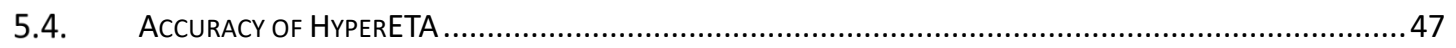

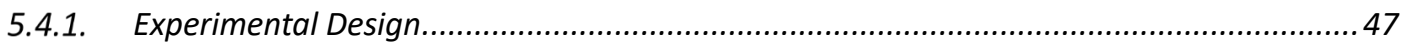

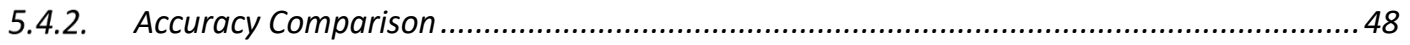

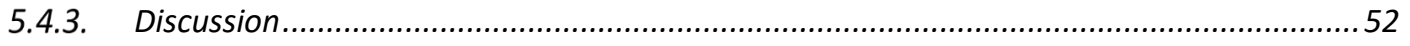

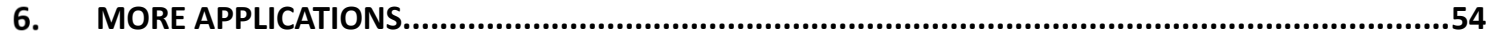

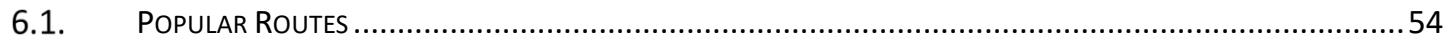




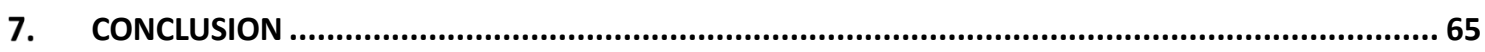

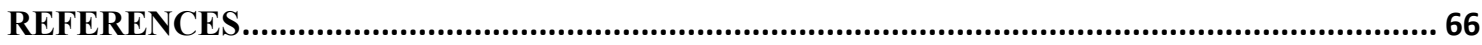

\section{LIST OF TABLES}

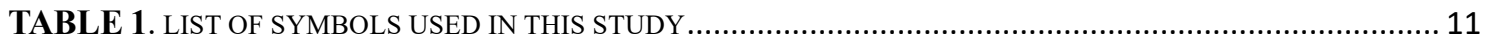

TABLE 2. COMPARISON OF HYPERCUBE AND GRID METHODS ..................................................... 47

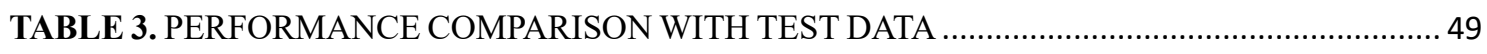

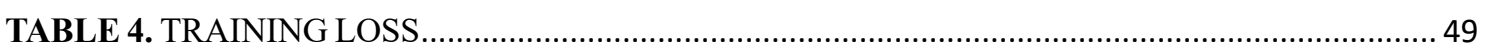

\section{LIST OF FIGURES}

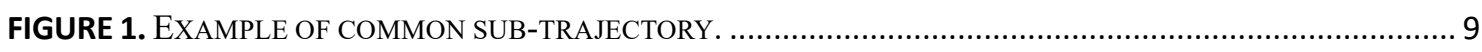

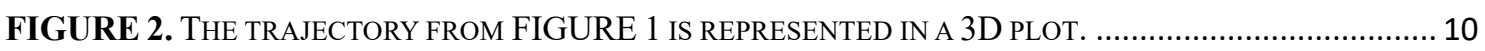

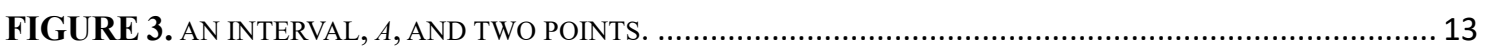

FIGURE 4. THE ONE DIMENSIONAL INTERVALS WHICH HAVE INTERSECTION. .......................................... 13

FIGURE 5. THE ONE-DIMENSIONAL INTERVALS WHICH HAVE INTERSECTION AND POINTS ON IT. ............... 14

FIGURE 6. AN EXAMPLE OF UNSTABLE POINTS. THE DASHED LINE IS A CUBE RANGE............................ 16

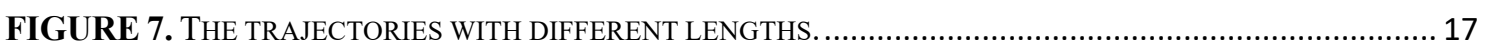

FIGURE 8. AN EXAMPLE OF A NOISY POINT. THE NOISY POINT IS ALONG AND AWAY FROM THE NORMAL

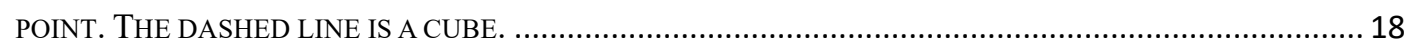

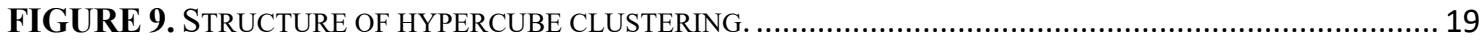

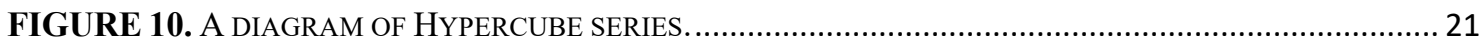

FIGURE 11. TWO TRAJECTORIES WITH AN INTERSECTION IN THE TIME DIMENSION. .............................. 23

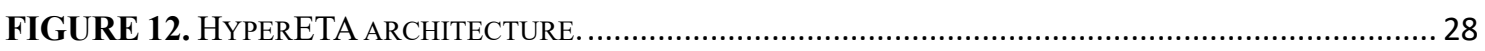

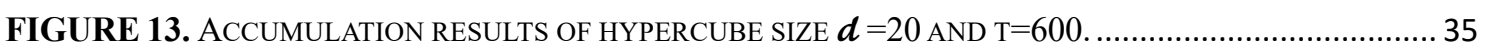

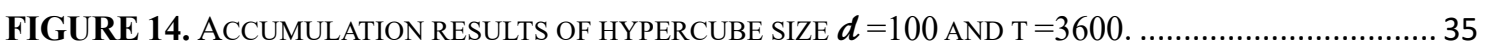

FIGURE 15. COMMON SUB-TRAJECTORY DISTANCES BY CLUSTERING UNDER VARIOUS PARAMETER COMBINATIONS

FIGURE 16. AN EXAMPLE THAT ILLUSTRATES THE DRAWBACK OF GRID CLUSTERING. TRAJECTORIES WITH SHORT GAPS LIE IN DIFFERENT BLOCKS ARE OVERLOOKED 38

FIGURE 17. The EXAMPle of HyPerCubes Clustering. TRAJECTORIES HAVE A VERY SHORT GAP, AND THE CUBES ARE OVERLAPPED EACH OTHER. THE DOT DASHED LINES ARE THE CUBE RANGE OF RED STAR SYMBOL “*”.

FIGURE 18. COMPUTATION TIME REQUIRED FOR HYPERCUBE CLUSTERING. ...................................... 40

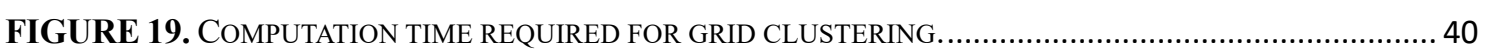


FIGURE 20. HYPERCUBES: NUMBER OF INTERSECTIONS TO BE CHECKED.

FIGURE 21. GRID VS. HyPERCUBES: MEMORY USAGE. “CUBE” REPRESENTS HYPERCUBE .42

FIGURE 22. QMEASURE OF HyPERCUBE, GRID, TRACLUS CLUSTERING. .44

FIGURE 23. DEVIATIONS IN HYPERCUBE CLUSTERING AND GRID CLUSTERING USING GEOGRAPHIC SIZE OF $100 \times 100 \mathrm{M}$

FIGURE 24. DEVIATIONS IN HYPERCUBE CLUSTERING AND GRID CLUSTERING USING GEOGRAPHIC SIZE OF $50 \times 50 \mathrm{M}$

FIGURE 25. DEVIATIONS IN HYPERCUBE CLUSTERING AND GRID CLUSTERING USING GEOGRAPHIC SIZE OF $25 \times 25 \mathrm{M}$ 46

FIGURE 26. ERROR RATES OF TRAJECTORIES HAVING DIFFERENT LENGTHS 51

FIGURE 27. RMSES OF TRAJECTORIES WITH DIFFERENT LENGTHS. 51

FIGURE 28. MAES OF TRAJECTORIES WITH DIFFERENT LENGTHS. 52

FIGURE 29. THE RESULT IS PRESENTED ON A TWO-DIMENSIONAL PLOT. THE BLACK DASHED LINE INDICATES THE 10\% MOST POPULAR SUB-TRAJECTORIES. THE GREEN DOTS INDICATE THE CENTRAL POSITION OF EACH HYPERCUBE

FIGURE 30. ZOOMED-IN VIEW OF RESULTS FROM THE PREVIOUS PLOT. THE BLACK DASHED LINES ARE FORMED BY A SERIES OF ARROWS TO SHOW THE ROUTE AND DIRECTION.....

FIGURE 31. POPULAR SUB-TRAJECTORIES WITH THE TEMPORAL DISTRIBUTION. THE BLACK DOTS INDICATE ARROWS, AND THE GREEN POINTS INDICATE THE CENTER OF HYPERCUBES, AS IN THE PREVIOUS FIGURE. THE THIRD DIMENSION (HEIGHT) IS TEMPORAL SPACE, RANGING FROM 20,000 SECONDS TO 120,000 SECONDS(5:30 A.M. TO NEXT DAY 9:20 A.M.). LONGITUDE RANGED FROM 116.2 TO 116.45. LATITUDE RANGED FROM 39.8 TO 40

FIGURE 32. RESULTS OBTAINED WITH $\mathrm{P}=0.5$. THE BLACK DASHED LINES INDICATE $50 \%$ MOST POPULAR SUB-TRAJECTORIES. THE GREEN DOTS INDICATE THE CENTRAL POSITION OF EACH HYPERCUBE. .......58

FIGURE 33. RESULTS OBTAINED WITH $\mathrm{P}=0.8$. THE BLACK DASHED LINES INDICATE $80 \%$ MOST POPULAR SUB-TRAJECTORIES. THE GREEN DOTS INDICATE THE CENTRAL POSITION OF EACH HYPERCUBE. .......59

FIGURE 34. HURRICANE TRAJECTORIES AND UNDERLYING TRENDS. ....................................................60

FIGURE 35. TEMPORAL DISTRIBUTION OF ALL HURRICANES INCLUDED IN THE RAW DATA. ....................61

FIGURE 36. TEMPORAL DISTRIBUTION OF HURRICANES BETWEEN DAYS 171 AND 200. THE DARK ARROWS INDICATE THE RESULTS OBTAINED USING HYPERCUBE CLUSTERING .62

FIGURE 37. TRENDS IN HURRICANE MOVEMENT WHEN DX $=10$ AND DY $=10 \ldots \ldots \ldots \ldots \ldots \ldots \ldots \ldots \ldots \ldots . . . \ldots 3$

FIGURE 38. TRENDS IN HURRICANE MOVEMENT WHEN DX $=2$ AND DY $=2$. 64 


\section{INTRODUCTION}

The purpose of trajectory clustering is to find similar trajectories. The purpose of common sub-trajectory clustering is to find similar parts on two trajectories. Trajectories can be a sequence of GPS(Global Positioning System) points or handwritten position marks. There are many applications for sub-trajectory clustering, e.g. identifying the trend of hurricanes[1] or finding optimum routes for ridesharing[2, 3].

$\ulcorner$ The following analysis is similar to the classical mathematical problem referred to as the traveling salesman problem. Note that this type of analysis generally requires a road network by which to map GPS trajectories. Alternative approaches (i.e., without a road network), such as Grid Clustering and TraClus[1], tend to suffer low efficiency and low accuracy. The theoretical time complexity of Grid Clustering is only $\mathrm{O}(1)$. The grid needs a vast array to store information. The initialization of the grid imposes significant time costs. The fact that grid clustering tends to overlook many common sub-trajectories significantly undermines accuracy. The low accuracy and computational speed of TraClus make it entirely unsuitable for large datasets. $\lrcorner$ [4]

$\ulcorner$ This study developed a novel scheme referred to as clustering $[4,5]$ to improve search performance. The proposed scheme was inspired by Orthogonal Range Searching in computational geometry [6]. The essential data structure is a Hypercube. A hypercube encapsulates a sub-trajectory. A Hypercube is shaped by longitude, latitude, time, and direction. The direction of a Hypercube is calculated according to the first and the last point within the Hypercube. Two Hypercubes intersect in the temporal and spatial domains and that have a similar direction indicate that two sub-trajectories are within a cluster. Initially, a trajectory is transformed into a series of Hypercubes. Therefore, clusters can be formed from the intersection of the Hypercubes. $\lrcorner[4]$ 
This study further applied Hypercubes Clustering to the estimated time of arrival (ETA) problem, which is a classic problem pertaining to travel planning, car navigation, and traffic dispatch. Numerous variables must be considered to predict the ETA accurately, and scholars have investigated different methods for improving the average accuracy of ETA predictions.

Several deep-learning-based methods have been proposed for solving the ETA problem [7-12]. A seminal method is DeepTTE [7], which uses historical trajectories as training data and predicts the ETA of a given trajectory. DeepTTE is a hybrid model [13] that integrates recurrent neural network and convolutional neural network $(\mathrm{CNN})$ models. It has been cited by many researchers, used for comparison, and extended.

Nevertheless, for the ETA problem, the existing deep learning techniques may not offer the best solutions for the following reasons. First, they require a considerable quantity of labeled training data. Second, they involve high computational costs, even for inference; hence, accelerators are required for computation, as indicated in [14] and [15]. Third, the accuracy of various deep learning methods can vary substantially, and this is the most critical problem. The severity of this phenomenon increases considerably when accelerators with poor arithmetic precision are used.

To address the aforementioned problems, This study presents a novel machinelearning algorithm called HyperETA, which can accurately predict the ETA of a given trajectory. In the training phase, HyperETA builds a trajectory model from historical trajectories. During the prediction phase, a given trajectory is transformed into hypercubes; the trajectory model is used to estimate the hypercubes' times, and the ETA is computed by summing up these times. Furthermore, in the proposed method, the parameters are set automatically, thus eliminating the need for guesswork or trial and error. Users can adjust the parameters by using meta-parameters. 
$\ulcorner$ Hypercube, TraClus, and grid clustering can be implemented without a road network, and identify common sub-trajectories from a set of trajectories. Performance comparison uses the GeoLife GPS trajectory dataset as test data. Hypercube clustering was shown to outperform grid clustering and TraClus in several aspects. $\lrcorner[4]$

This study conducted experiments to compare HyperETA with a deep-learningbased method called DeepTTE by using Cheng-Du taxi trajectories as a benchmark. The results demonstrated that HyperETA outperformed DeepTTE in terms of prediction accuracy.

The contributions of this study are listed the following:

1. This study proposes a novel common sub-trajectory retrieval method with directionality, called Hypercube Clustering.

2. Hypercube Clustering is applied to a new ETA method, called HyperETA, that was designed in this study.

3. An algorithm for automatically setting parameters is designed.

4. This study conducted experiments using real-world datasets comprising GPS points, including Geolife dataset and the dataset generated by taxis in Cheng-du.

5. Hypercubes Clustering outperforms grid clustering, a classical method, and TraClus. a famous trajectory clustering method.

6. HyperETA outperforms DeepTTE, a popular deep-learning-based method.

7. A few problems associated with deep learning are discussed, including data size, GPU's precision, training loss, and low accuracy.

8. Two extra applications to demonstrate the usages of Hypercubes Clustering. 


\section{LITERATURE REVIEW}

This section discusses previous studies related to trajectory clustering and predicting ETA.

\subsection{Computational Geometry}

$\ulcorner$ The rectangle intersection problem has been thoroughly investigated in computational geometry [6]. Buchin et al. [16] used computational geometry to get similar portions of a given trajectory, it also consider temporal attribute. They employed time-shifting to synchronize trajectories, and their method can also be used to find common sub-trajectories. In some situations, this method can be implemented in linear time as long as the duration is fixed. However, this method tends to be somewhat inefficient when applied to large sets of trajectories with numerous vertices. $\lrcorner[4]$

\subsection{Methods of Finding Common Trajectory}

$\ulcorner$ Methods based on curve similarities, such as Longest Common Sub-Sequence LCSS[17], Edit Distance on Real sequence(EDR)[18], Hausdorff distance[19], and Dynamic Time Warping(DTW)[20, 21], were not intended for multiple common subtrajectories. Those methods can be used to measure the shape similarity of two trajectories; however, those methods cannot be used to find common sub-trajectories. The computational cost of this method is high due to the direct processing of GPS points without a points-reduction method. These methods are fragile by noisy data, which can result in huge Euclidean distances and eventual breakdown. Sub-trajectory clustering [1, 22] divides a trajectory into line segments; however, the computational time of finding common sub-trajectories can be high when handling intricate lines. Most of these methods consider only spatial data but spatiotemporal data [23]. A recent method, ensemble clustering, have proven effective [24] in image clustering, but not trajectory 
clustering. One data clustering method designed specifically for trajectory clustering is TraClus [1]. TraClus is a famous classical method in trajectory clustering[25-27], which is evaluated in this study. $\lrcorner[4]$

\subsection{Graph-based Methods}

$\ulcorner$ Graph-based methods, such as Network Hausdorff Distance(NHD) [28, 29], grid with road network information method [30], and road network and feature vector [31], use road network information to simplify the analysis of trajectories. Road network information is alike to the nodes and the edges in the traveling salesman problem (TSP). Unfortunately, these methods function well only when they have the newest road network information, thereby necessitating frequent network updates. Nonetheless, graph-based methods are convenient. Reducing the trajectory into a graph makes it possible to use approaches based on graph theory. This study aims to develop a method that performs well without depending on a road network. $\lrcorner[4]$

\subsection{Ridesharing System and Grid Clustering}

$\ulcorner$ Previous studies on ridesharing systems[32] have enjoyed a good deal of success. Nonetheless, taxi ridesharing and salient traffic problems also require road network information. The system[3] splits user trajectories into a number of segments (based on temporal distance) and matches them within a grid space of grid clustering. Grid clustering $[3,33]$ is a well-known sub-trajectory clustering method in which trajectories are segmented within a grid space by checking cells through which traces pass in order to derive a common sub-trajectory. Common sub-trajectories can be retrieved within the constant time in theory. However, the tendency of grid clustering to overlook many common sub-trajectories can lead to poor results. The paths through a particular block are regarded as potential common sub-trajectories, whereas the trajectories through 
adjacent blocks are not, even when the gap between two paths is only a few centimeters.

\subsection{Deep Learning Methods}

Deep learning, a relatively new paradigm in artificial intelligence, has attracted substantial attention from the research community owing to its remarkable potential compared with traditional techniques. Compared with traditional machine learning (ML) methods, deep learning can be used to model sophisticated functions through layers of nonlinear transformations trainable from the beginning to the end. Methods based on deep learning models have considerably advanced state of the art in diverse domains. The success of deep learning has attracted the attention of researchers from different domains, who have explored its use for solving various problems.

Deep neural networks (DNNs) that combine artificial neural networks and Deep learning are considered state-of-the-art among ML techniques [34]. DNNs have been applied in many domains[35]. For example, NVIDIA's researchers used DNNs in hand gesture recognition [36]; they used their super-advanced machine, four super GPU cards, and the latest library they developed for deep learning.

Training DNNs to obtain a good model involves stringent requirements. DNNs often require vast sources of data to learn appropriate abstractions[35]. Consequently, massive computing power is required for data processing. Accordingly, expensive GPUs and compatible mainframes are required. Such high levels of computing power are essential for tuning the parameters that define deep learning models, such as learning rates, the number of neural network layers, and network architecture. The parameters are determined intuitively and through trial-and-error approaches; they must be determined as fast as possible. 
One limitation of DNNs is their high level of opacity [34]. The multilayer mathematical neuronal structure of DNNs makes it difficult to interpret them (loosely defined as the science of comprehending what a model does[37]) and explain why certain inputs lead to certain outputs. Therefore, DNNs are typically executed as black boxes[38] due to this lack of transparency.

Several studies have used deep learning to forecast travel times. For example, [7] used geo-convolutional neural networks in DeepTTE. DeepTTE has been cited by many researchers, in addition to being compared with and extended [8] to predict bus travel times.

Graph convolutional neural networks (GCNNs) have been widely used to predict ETA [9-12], where "Graph" represents the required roadmaps. All of these methods accept spatiotemporal data. In addition, a GCNN-based origin-destination method [9] was proposed for ETA estimation. An origin-destination method is not concerned with paths. Instead, it aims to determine ETA by depending only on two points. In [10], deep learning techniques such as GCNN, long short-term memory (LSTM), and gated recurrent unit (GRU) were used to predict ETA. In [11], a hybrid spatiotemporal GCNN was used to predict ETA and possible congestion. The experimental dataset was not published online. In [12], a multilayer perceptron (MLP) was used to predict ETA.

A GCNN requires road network information to reduce the ETA to some graph problem, such as the traveling salesman problem (TSP). Such a requirement may not be achievable in many applications. The present study focused on methods that do not use road network information. Therefore, GCNN methods were not considered in this study.

For most of the papers published thus far, the software has not been published online, and specifications of the computer language and hardware environment have not been provided. In some of these studies, the experimental data used are not freely 
available. Therefore, these data could not be involved in our experiments. Some of the works require roadmaps, which is not the focus of the proposed method.

\subsection{The classical clustering methods}

There are a lot of classical clustering methods such as K-means[39] and DBSCAN[40] that seen like can be adapt to the problems in this study. DBSCAN is a density-based clustering algorithm. DBSCAN can find arbitrarily shaped clusters. kmeans clustering is to partition $\mathrm{n}$ observations into $\mathrm{k}$ clusters in which each observation belongs to the cluster with the nearest mean. However, those methods didn't help the application in this study while they just get a huge block(cluster) by grouping every trajectory that has parts similar. What this study needs are fixed-range and high-density segments to draw a picture like which in section 6.2 or estimate the drive speed of each road segment. Using K-means needs to decide "K" but not the method in this study. It is possible to decide "K" by the "converge" or some extra optimization algorithms. Despite they will make the method in this study too complicate to be used and slow the performance, the clusters are not what this study needs. The method in this study do not need to decide "K" and can not be decided by "K". Even if I redesign those methods, add some details that I design in a program, it may fit the requirements in this study. But, they will become different methods and it exceeds my research domain. And, they will become "my" methods. 


\section{DEFINITIONS OF COMMON SUB-TRAJECTORIES}

$\ulcorner$ FIGURE 1 presents an example of common sub-trajectory in which each symbol represents a GPS point. Two trajectories (the main track and the track 73) are identified as having a common sub- trajectory relationship. Two sub- trajectories are within the range of the red circle. The average distance (spatial gap) between the two paths within the red circle is approximately 20 meters. $\lrcorner$

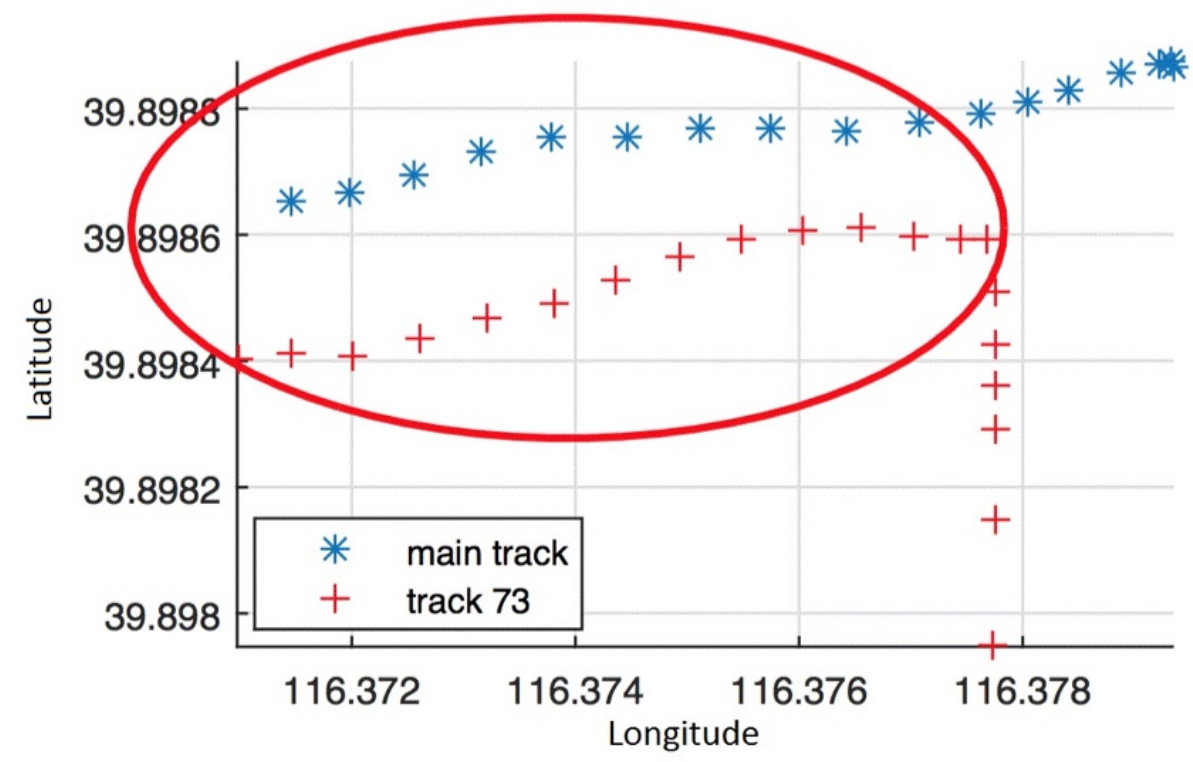

FIGURE 1. Example of common sub-trajectory. 
$\ulcorner$ FIGURE 2 shows the same trajectory in a three-dimensional (3D) space with temporal space as the vertical axis. This is also known as a three-dimensional Space-time continuum in the special relativity of physics. $\lrcorner[4]$

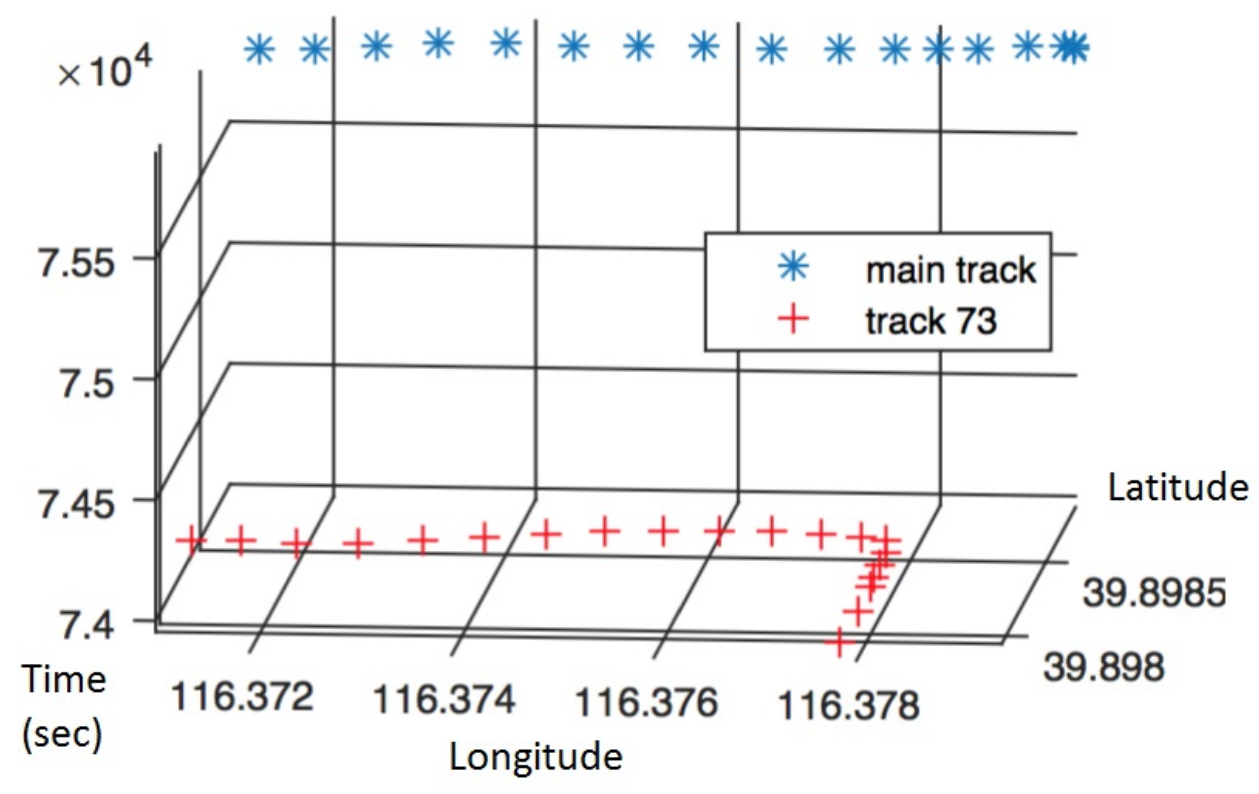

FIGURE 2. The trajectory from FIGURE 1 is represented in a 3D plot. 
TABLE 1. list of symbols used in this study

\begin{tabular}{cl}
\hline Symbol & Description \\
\hline \hline $\boldsymbol{T}$ & A trajectory \\
\hline$p_{i}$ & $\begin{array}{l}\text { A point in a trajectory. Including three attributes, } x_{i}, y_{i}, t_{i}, \text { which are } \\
\text { longitude, latitude, timestamp, respectively. }\end{array}$ \\
\hline$p_{i} \cdot x_{i}$ & Longitude of $p_{i}$ \\
\hline$p_{i} \cdot y_{i}$ & Latitude of $p_{i}$ \\
\hline$p_{i} \cdot t_{i}$ & Timestamp of $p_{i}$ \\
\hline$d_{x}$ & longitude length. It can be measured in meter or longitude difference. \\
\hline$d_{y}$ & latitude length. It can be measured in meter or latitude difference. \\
\hline$\tau$ & time length \\
\hline$d$ & Geographical length. It is measured in meter or the difference of \\
\hline $\boldsymbol{C}$ & coordinate. \\
\hline$c_{i}$ & A cubes sequence. $\boldsymbol{C}=\left\{c_{1}, c_{2}, \cdots, c_{i}, \cdots\right\}$ \\
\hline$c_{i} \cdot x$ & Longitude of $c_{i}$ \\
\hline$c_{i} \cdot y$ & Latitude of $c_{i}$ \\
\hline$c_{i} \cdot t$ & Timestamp of $c_{i}$ \\
\hline$c_{i} \cdot \theta$ & Direction of $c_{i}$ \\
\hline$\Phi$ & The minimal acceptable angle difference. \\
\hline$p$ & A percentage to decide $\mu$. \\
\hline$\mu$ & The minimal degree of a vertex. \\
\hline
\end{tabular}

$\ulcorner$ TABLE 1 lists a number of important symbols used in this paper. Trajectory $T$ is defined as a sequence of trajectory points, as follows:

$$
T=\left\{p_{1}, p_{2}, p_{3}, \cdots, p_{n}\right\}
$$

where $n$ is the number of trajectory points in $T$. Each trajectory point $p_{i}=\left\{x_{i}, y_{i}, t_{i}\right\}$ consists of three attributes: $x_{i}$ for longitude, $y_{i}$ for latitude, and $t_{i}$ for timestamp. This 
study use $p_{i} \cdot x, p_{i} \cdot y$, and $p_{i} . t$ to represent the latitude, longitude, and timestamp of point $p_{i}$, respectively. Trajectory $T$ can be partitioned into subsequences, as follows:

$$
T=\left\{s_{1}, s_{2}, \cdots, s_{k}\right\}
$$

in which $s_{i}$ denotes the $i$-th sub-trajectory of $\left.T.\right\lrcorner \quad[4]$

Two sub-trajectories, $s_{i}=\left\{p_{1}, p_{2}, \cdots, p_{n_{i}}\right\}$ and $s_{j}=\left\{p_{1}^{\prime}, p_{2}^{\prime}, \cdots, p_{n_{J}}^{\prime}\right\}$, are referred to as "common sub-trajectories" if the set of trajectory points , $P=$ $\left\{p_{1}, p_{2}, \cdots, p_{n_{i}}, p_{1}^{\prime}, p_{2}^{\prime}, \cdots, p_{n_{J}}^{\prime}\right\}$, satisfies the following three conditions:

$$
\begin{aligned}
& \max _{p \in P}(p \cdot x)-\min _{p \in P}(p \cdot x) \leq d_{x} \\
& \max _{p \in P}(p \cdot y)-\min _{p \in P}(p \cdot y) \leq d_{y} \\
& \max _{p \in P}(p \cdot t)-\min _{p \in P}(p \cdot t) \leq \tau
\end{aligned}
$$

\subsection{Theorem}

Hypercubes clustering uses the cubes intersection to identify common subtrajectories. To prove the idea and to show the maximal distance between the common sub-trajectories, This study shows the theoretical result in Theorem 1. The theorem is based on Lemma 1 and Lemma 2. The theorem is in one dimension, but it can be inferred to the cubes intersection while each dimension is examined separately. Furthermore, the theorem considers only two points in an interval and ignores other points between the two. It is because the distance between the endpoints of different intervals is the maximal distance between two sub-trajectories. The theorem shows that the distance between the common sub-trajectories must be smaller than $\frac{3}{2}$ cube size. 


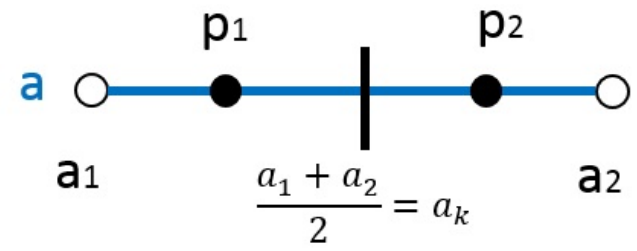

FIGURE 3. an interval, $a$, and two points.

LEMMA 1. Given an interval $a$ from $a_{1}$ to $a_{2}$, and two points as FIGURE 3 . It is defined that $p_{1}$ is the left point and $p_{2}$ is the right point. $a_{k}=\frac{a_{1}+a_{2}}{2}$, based on (5). If $a_{k}=\frac{p_{1}+p_{2}}{2}$, then $p_{1} \leq a_{k}$ and $p_{2} \geq a_{k}$.

PROOF. WLOG, $p_{1} \leq p_{2}$, and $\frac{p_{1}+p_{2}}{2}=a_{k} \Rightarrow p_{1}+p_{2}=2 \cdot a_{k}$

- $\quad p_{1} \leq p_{2} \Rightarrow p_{1}+p_{1} \leq p_{2}+p_{1}=2 \cdot a_{k} \Rightarrow 2 \cdot p_{1} \leq 2 \cdot a_{k} \Rightarrow p_{1} \leq a_{k}$

- $\quad p_{1} \leq p_{2} \Rightarrow p_{1}+p_{2} \leq p_{2}+p_{2}=2 \cdot p_{2} \Rightarrow 2 \cdot a_{k} \leq 2 \cdot p_{2} \Rightarrow a_{k} \leq p_{2}$

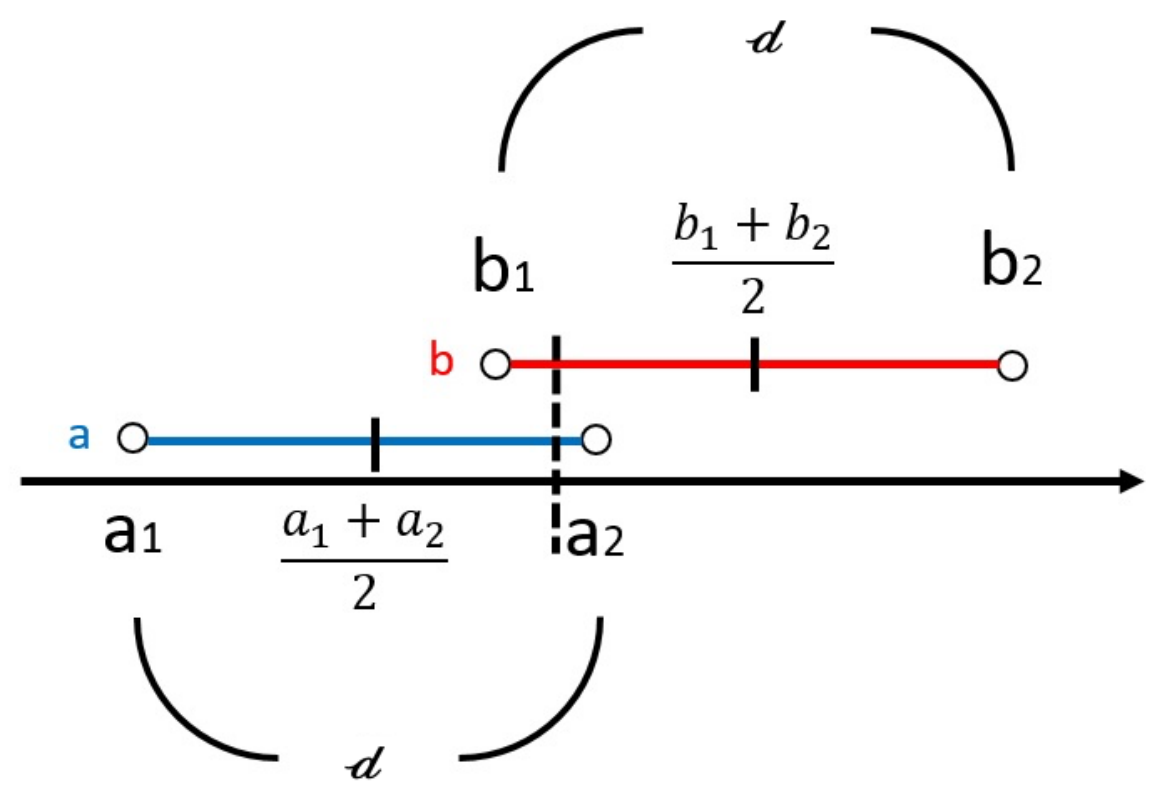

FIGURE 4. The one dimensional intervals which have intersection. 
LEMMA 2. Given two intervals, the left interval $a$ and the right interval $b$, both with lengths $d$ as FIGURE 4. If two intervals have intersection, the distance between two centers in each interval will be smaller than $d$.

PROOF. WLOG, $a_{1} \leq b_{1}, a_{2} \leq b_{2}$.

It is known that $\left(a_{1}, a_{2}\right) \cap\left(b_{1}, b_{2}\right) \neq \varnothing$

$\Rightarrow b_{1}<a_{2}=a_{1}+\epsilon \Rightarrow b_{1}-a_{1}<d$

Also, $b_{2=} b_{1}+d$ is known.

The distance between two centers in each interval

$=\frac{b_{1}+b_{2}}{2}-\frac{a_{1}+a_{2}}{2}=\frac{b_{1}+b_{2}+d}{2}-\frac{a_{1}+a_{2}+d}{2}=b_{1}-a_{1}<d$
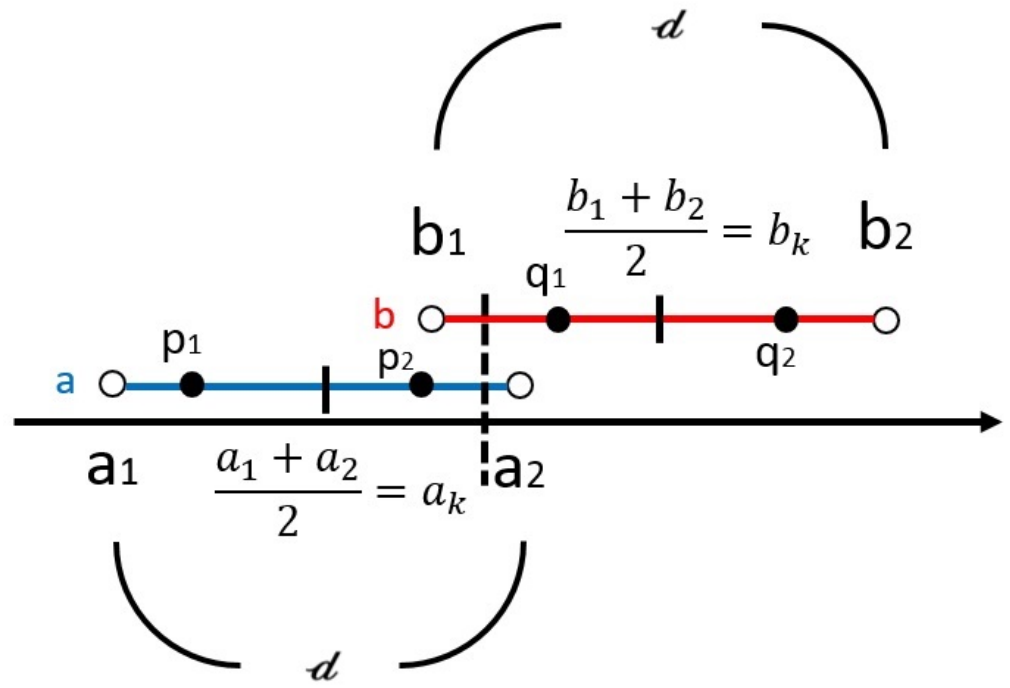

FIGURE 5. The one-dimensional intervals which have intersection and points on it.

THEOREM 1. Given two intervals, the left interval $a$ and the right interval $b$, both with lengths $\varepsilon$ as FIGURE 5. Each interval has two points in it. The left point $p_{1}$ and the right point $p_{2}$ are in $a$. The left point $q_{1}$ and the right point $q_{2}$ are in $b$. If two intervals have intersection, then $\left|p_{1}-q_{1}\right|<\frac{3}{2} d$ and $\left|p_{2}-q_{2}\right|<\frac{3}{2} d$.

ROOF. It is known that

1) WLOG, $b_{1} \geq a_{1} \Rightarrow a_{1}-b_{1} \leq 0$. 
2) $\left(a_{1}, a_{2}\right) \cap\left(b_{1}, b_{2}\right) \neq \varnothing \rightarrow$ LEMMA 2: $b_{1}-a_{1}<d$

3) $\left\{\begin{array}{l}\frac{p_{1}+p_{2}}{2}=\frac{a_{1}+a_{2}}{2}=a_{k} \\ \frac{q_{1}+q_{2}}{2}=\frac{b_{1}+b_{2}}{2}=b_{k}\end{array} \Rightarrow\right.$ LEMMA 1, $\Rightarrow \begin{array}{l}a_{1}<p_{1} \leq a_{k} \\ b_{1}<q_{1} \leq b_{k}\end{array}$

$p_{1}-q_{1} \leq a_{k}-b_{1}=a_{1}-b_{1}+\frac{d}{2} \leq \frac{d}{2}$

$q_{1}-p_{1} \leq b_{k}-a_{1}=b_{1}-a_{1}+\frac{\varepsilon}{2} \leq \frac{3}{2} d$

$\left|p_{1}-q_{1}\right| \leq \max \left(p_{1}-q_{1}, q_{1}-p_{1}\right)<\frac{3}{2} d$

It is known that

1) WLOG, $b_{2} \geq a_{2} \Rightarrow a_{2}-b_{2} \leq 0$.

2) $\left(a_{1}, a_{2}\right) \cap\left(b_{1}, b_{2}\right) \neq \emptyset \rightarrow$ LEMMA 2: $b_{2}-a_{2}<d$

3) $\left\{\begin{array}{l}\frac{p_{1}+p_{2}}{2}=\frac{a_{1}+a_{2}}{2}=a_{k} \\ \frac{q_{1}+q_{2}}{2}=\frac{b_{1}+b_{2}}{2}=b_{k}\end{array} \Rightarrow \operatorname{LEMMA~1,~} \Rightarrow \begin{array}{l}a_{k}<p_{2} \leq a_{2} \\ b_{k}<q_{2} \leq b_{2}\end{array}\right.$

$p_{2}-q_{2} \leq a_{2}-b_{k}=a_{2}-b_{2}+\frac{d}{2} \leq \frac{d}{2}$

$q_{2}-p_{2} \leq b_{2}-a_{k}=b_{2}-a_{2}+\frac{d}{2} \leq \frac{3}{2} d$

$\left|p_{2}-q_{2}\right| \leq \max \left(p_{2}-q_{2}, q_{2}-p_{2}\right)<\frac{3}{2} d$

\subsection{Issues}

There are several issues when processing trajectory data comparing to other types of data. Some of them are addressed in [17]. Here I summarized them with four issues.

The alignment problems - These problems are caused when one trajectory is aligned with another. A trajectory can vary in the shape of the distribution and can vary different from other trajectories but still have similar parts. The differences are such as non-fixed points interval, the unstable trajectory, and different lengths. First, the non- 
fixed points interval is that the interval of points in a trajectory usually is not the same; it also happens between two trajectories. This phenomenon is caused by different sampling rates or different moving speeds; there are various sampling rates on various GPS devices. For example, the moving speed of a car is different from walking. FIGURE 17 shows an example of the different moving speeds of two trajectories. Another way of non-fixed points interval is a huge unexpected interval; it is caused by a failed sensor or blocked signal. It is also caused a significant problem when aligning trajectories. Second, the unstable trajectory, it is looked like a wave even if the GPS device moves in a straight line, where it is caused by the inaccuracy of sensing devices. FIGURE 6. An example of unstable points. The dashed line is a cube range. shows an example of an unstable trajectory. Usually, the trajectory will be smoothed for better results. Third, the various lengths are that the length of a trajectory is different from another. One of the reasons for this phenomenon is the various lengths of sampling time; a GPS device is started longer than others. This phenomenon will cause the alignment problem in which the first points or the last points of two trajectories shell be aligned. FIGURE 7 shows a simple example of this phenomenon.

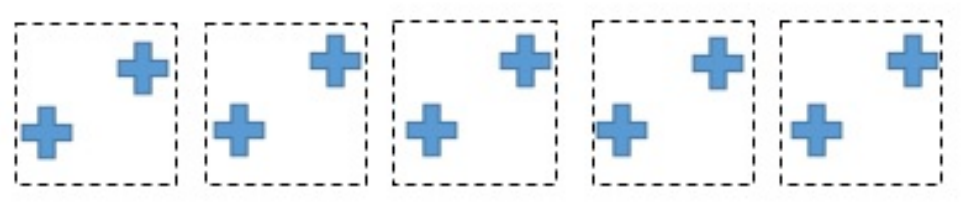

FIGURE 6. An example of unstable points. The dashed line is a cube range. 


\section{+t+t++t+t+t}

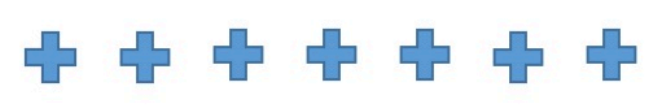

FIGURE 7. The trajectories with different lengths.

Similar trajectories in different locations - Two trajectories have a similar curve but appear in different locations on a map. For example, two parallel trajectories pass through different roads, and they are similar because the gap distance between the paralleled roads is smaller than a threshold. In the ridesharing problem, the user of two trajectories can share a ride simply by one user walk to another road. In sign language recognition, this situation is also a common problem. This situation also happens in the time domain; two trajectories have the same moving paths but appear at different time. This issue is much difficult when finding partially similar sub-trajectories. FIGURE 1 shows an example.

The outliner - A few trajectory points are appeared in an unreasonable position, a jerky movement, which happens at abnormal sensor function. These noisy causes the result failed while the Euclidean distance is very long between the noisy points and the normal points. The major difference between the noisy and the normal trajectory points is that the number of noise points is few and easily observed as outliers. FIGURE 8 shows an example of noise. 


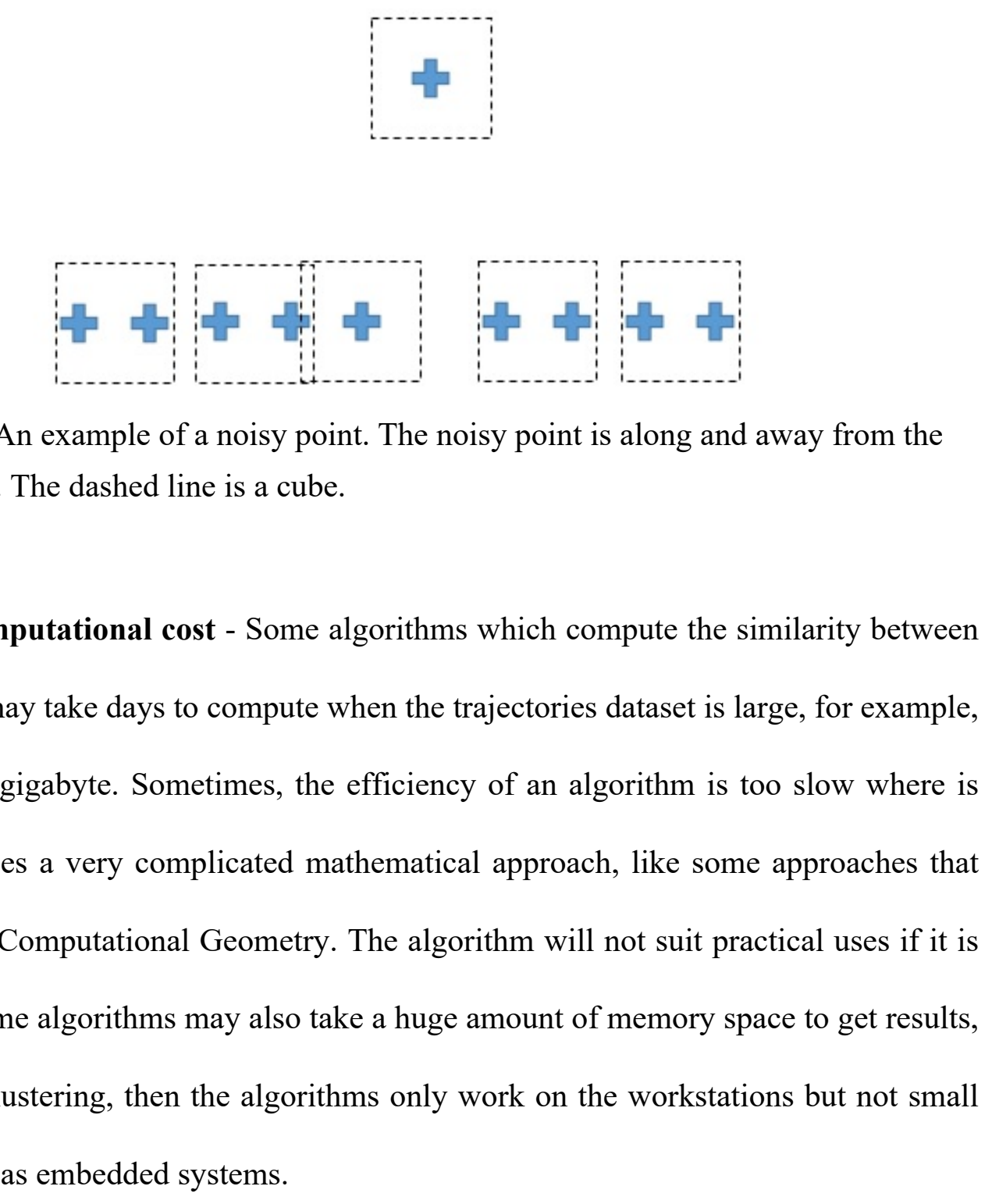

FIGURE 8. An example of a noisy point. The noisy point is along and away from the normal point. The dashed line is a cube.

Computational cost - Some algorithms which compute the similarity between trajectories may take days to compute when the trajectories dataset is large, for example, more than a gigabyte. Sometimes, the efficiency of an algorithm is too slow where is because it uses a very complicated mathematical approach, like some approaches that extend from Computational Geometry. The algorithm will not suit practical uses if it is too slow. Some algorithms may also take a huge amount of memory space to get results, as the grid clustering, then the algorithms only work on the workstations but not small devices such as embedded systems. 


\section{ALGORITHMS}

This section outlines $\ulcorner$ the details of the proposed Hypercube-clustering algorithm. It is presented in the form of pseudo-code for implementation in MATLAB and $\mathrm{C}++$. The algorithm proceeds through three steps: 1) preprocessing raw data, 2) Hypercube-intersection, and 3) clustering. Each step is illustrated in the following subsections. $\lrcorner[4]$

The structure of Hypercube clustering is presented in FIGURE 9.

Trajectories dataseet

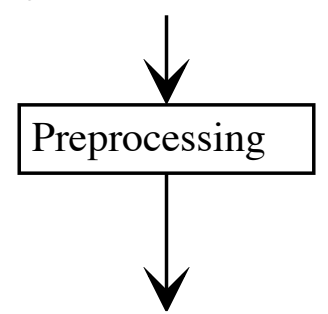

Multiple hypercubes sequences

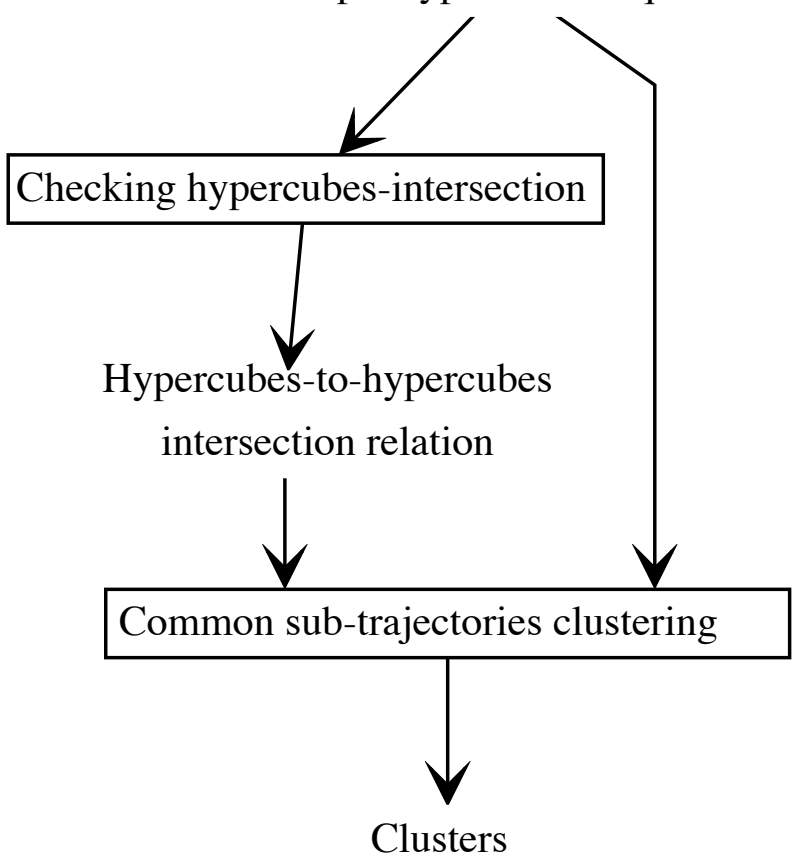

FIGURE 9. Structure of hypercube clustering. 


\subsection{Preprocessing}

$\ulcorner$ In this step, a trajectory is transformed into a Hypercube sequence, and each Hypercube includes part of the trajectory. The points of the trajectory are sequentially scanned and grouped based on the range $\left(d_{x}, d_{y}\right.$, and $\left.\tau\right)$ of a Hypercube. The central position of a Hypercube is the center of the inside points. The direction of a Hypercube is computed according to the first and the last point within the Hypercube. The time complexity with this step is $\mathrm{O}(\mathbf{n})$, where $\mathbf{n}$ is the number of GPS points. $\lrcorner$

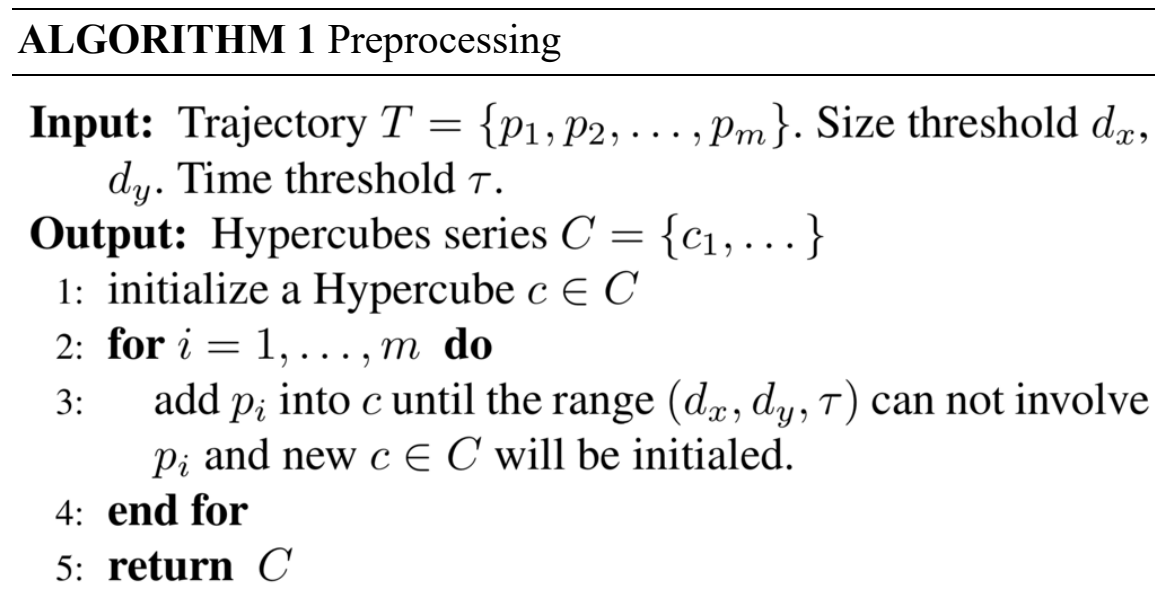

$\ulcorner$ ALGORITHM 1 outlines the details of this transformation. Input $T$ is a trajectory in which $p_{i} . t$ is always larger than $p_{i-1} . t$, which is ordered temporally; $d_{x}$ and $d_{y}$ indicate the geographic size of a Hypercube; and $\tau$ is the temporal size. Initially, $c_{1}$ is created, and then trajectory points are sequentially inserted into $c_{j}$. A new Hypercube is created if one of the criteria in (4) the range $\left(d_{x}, d_{y}\right.$, and $\left.\tau\right)$ is violated by the newly inserted $p_{i \cdot\lrcorner[4]}$ 


$$
\begin{aligned}
& \max _{p \in c_{j}}\left|p_{i} \cdot x-p \cdot x\right| \leq \epsilon_{x} \\
& \max _{p \in c_{j}}\left|p_{i} \cdot y-p \cdot y\right| \leq \epsilon_{y} \\
& \max _{p \in c_{j}}\left|p_{i} \cdot t-p . t\right| \leq \tau .
\end{aligned}
$$

The center and the direction of $c_{j}$ must be determined before the next Hypercube is created or at the end of period T. Let $\left(c_{j} \cdot x, c_{j} \cdot y, c_{j} \cdot t\right)$ be the center of $c_{j}$, the formula of which is as (5):

$$
\begin{aligned}
c_{j} . x & =\frac{\max _{p \in c_{j}} p \cdot x+\min _{p \in c_{j}} p \cdot x}{2} \\
c_{j} . y & =\frac{\max _{p \in c_{j}} p \cdot y+\min _{p \in c_{j}} p \cdot y}{2} \\
c_{j} . t & =\frac{\max _{p \in c_{j}} p \cdot t+\min _{p \in c_{j}} p \cdot t}{2} .
\end{aligned}
$$

The direction of Hypercube $c_{j}$ is represented by angle $c_{j} . \theta$, the definition of which is (6), and $p_{1}$ and $p_{n}$ are respectively the first and last points inserted into $c_{j}$.

$$
c_{j} \cdot \theta=\arctan \left(\frac{p_{n} \cdot y-p_{1} \cdot y}{p_{n} \cdot x-p_{1} \cdot x}\right)
$$

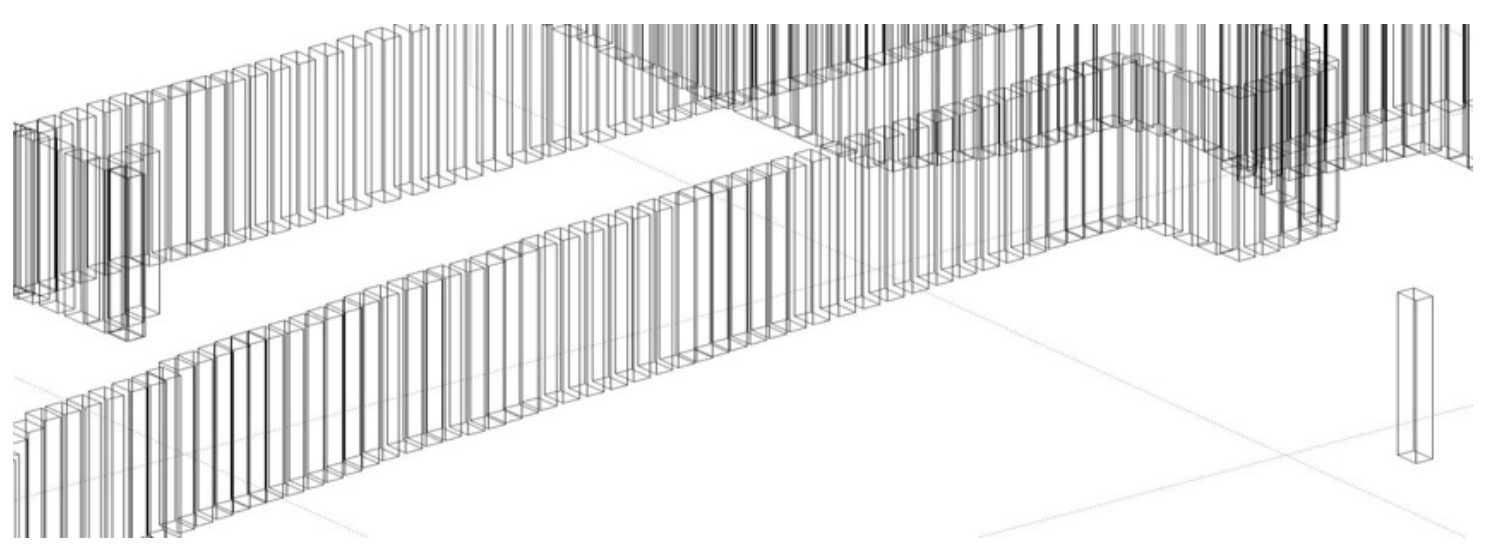

FIGURE 10. A diagram of Hypercube series. 
FIGURE 10 is a visualization of cubes following transformation. This study draws the range of $\left(d_{x}, d_{y}\right.$, and $\left.\tau\right)$, and it looks like cubes, which is the reason for the name. With the fourth dimension, the direction, the Hypercube has been named.

$\ulcorner$ This step is meant to prevent some basic problems associated with processing trajectories. For non-fixed point intervals, this step turns the points into fixed range Hypercubes. This step can also reduce computational costs by reducing the number of objects to be computed. $\lrcorner[4]$

It is recommended that beginner users adopt an $\varepsilon$ value equal to the size of a view divide by 20 . Note that the view is also the area of the trajectories in these experiments.

\subsection{Hypercube Intersections}

$\ulcorner$ This step checks the intersection relation between two Hypercube sequences in four dimensions. First, the algorithm checks the intersection between two sequences in temporal space. If there is no overlapping in temporal space, there will be no intersection, and the checking only costs constant time. Further examination begins at a temporal intersection if there is one. The examination includes temporal intersections, geographic intersections, and similarity in the relative direction of Hypercubes. The average time complexity with this step is $\mathrm{O}(\mathbf{n})$, where $\mathbf{n}$ is the number of Hypercubes. $\lrcorner[4]$ 


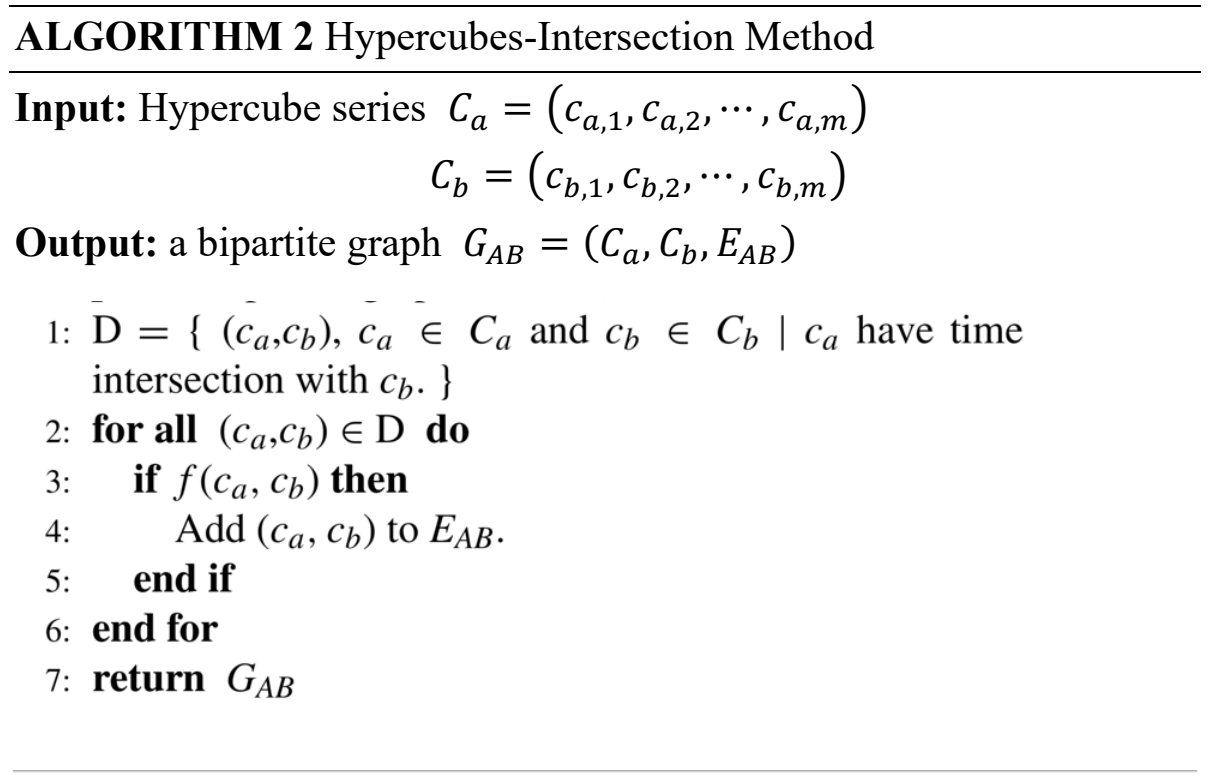

$\ulcorner$ ALGORITHM 2 shows the Hypercubes-Intersection method. If there is no temporal intersection, an empty set is output and only cost constant time complexity. The input is two sequences, $C_{a}$ and $C_{b}$ (in temporal order) and the output is information pertaining to the intersection. Lines 1 check whether $C_{a}$ and $C_{b}$ have intersections in the time domain. $\lrcorner[4]$

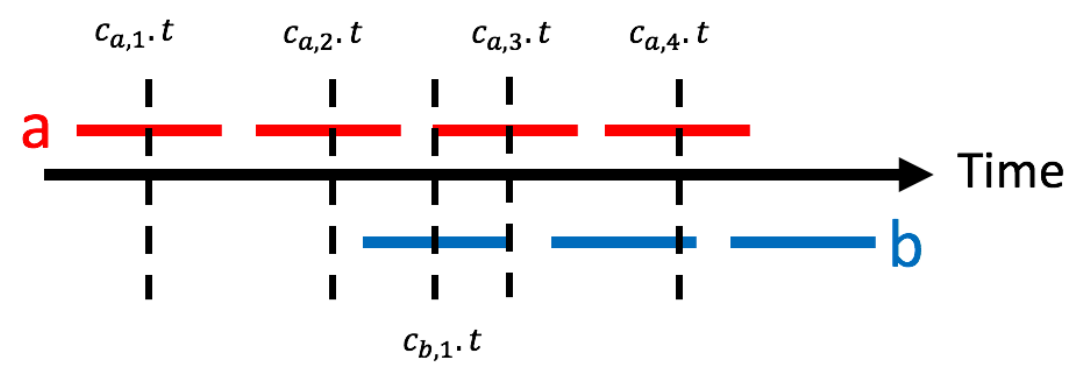

FIGURE 11. Two trajectories with an intersection in the time dimension.

$\ulcorner$ FIGURE 11 presents an example of a temporal intersection. If there is a temporal intersection, then the algorithm uses the fact that the points in a trajectory are always in ascending temporal order so as to accelerate the checking process. The 
algorithm sequentially checks the temporal intersections between Hypercubes $\lrcorner$ [4]. A simple experiment that comparing this time-sequence technique with no time-sequence technique has been done and used Geolife dataset as a benchmark. Both methods got exactly the same result but different time cost. The no time-sequence technique edition used 1189 seconds to find every intersection. The time-sequence technique edition used only 425 seconds. The time-sequence technique edition is 2.8 times faster. The worse case time complexity of this technique is still $\mathrm{O}\left(n^{2}\right)$, where $\mathrm{n}$ is a number of cubes. However, the best case is $\mathrm{O}(1)$.

$\ulcorner$ Only Hypercubes with temporal intersections are checked for geographic intersections and having a similar direction. (7) is used to check two temporallyintersected Hypercubes, $c_{i}$ and $c_{j}$, in terms of geographic intersections and directional intersections. For direction checking, this study defines that two sub-trajectories have no intersection relation if their difference in angle exceeds threshold $\Phi\lrcorner.[4]$

$$
f\left(c_{i}, c_{j}\right)= \begin{cases} & \left|c_{i} \cdot x-c_{j} \cdot x\right|<\epsilon_{x} \text { and } \\ \text { true }, & \left|c_{i} \cdot y-c_{j} \cdot y\right|<\epsilon_{y} \text { and } \\ & \angle\left(c_{i} \cdot \theta, c_{j} \cdot \theta\right)<\phi \\ \text { false, }, & \text { otherwise. }\end{cases}
$$

$\ulcorner$ The output is information related to Hypercube intersections, which are stored in a bipartite graph data structure, $G_{A B}=\left(\mathrm{A}, \mathrm{B}, \mathrm{E}_{\mathrm{AB}}\right)$, where each vertex in $\mathrm{A}$ is a Hypercube in $c_{a}$, each vertex in $\mathrm{B}$ is a Hypercube in $c_{b}$, and $\mathrm{E}_{\mathrm{AB}}=\left\{\left(c_{a}, c_{b}\right) \mid c_{a} \in\right.$ $C_{a}$ and $c_{b} \in C_{b}$. Each edge in $G_{A B}$ represents a pair of common sub-trajectories; because with proper normalization, it can be shown that two sub-trajectories satisfy the three conditions of common sub-trajectories, given in (1),(2) and (3). $\lrcorner[4]$ 


\subsection{Common Sub-trajectories Clustering}

$\ulcorner$ In this stage, the Hypercubes are grouped. A Hypercube is grouped with other Hypercubes with which it shares an intersection. The grouped Hypercubes are then removed from the data on Hypercube intersections. This process is repeated until every remaining Hypercube has a number of intersections that smaller than a threshold. The algorithm is as ALGORITHM 3. $\lrcorner[4]$

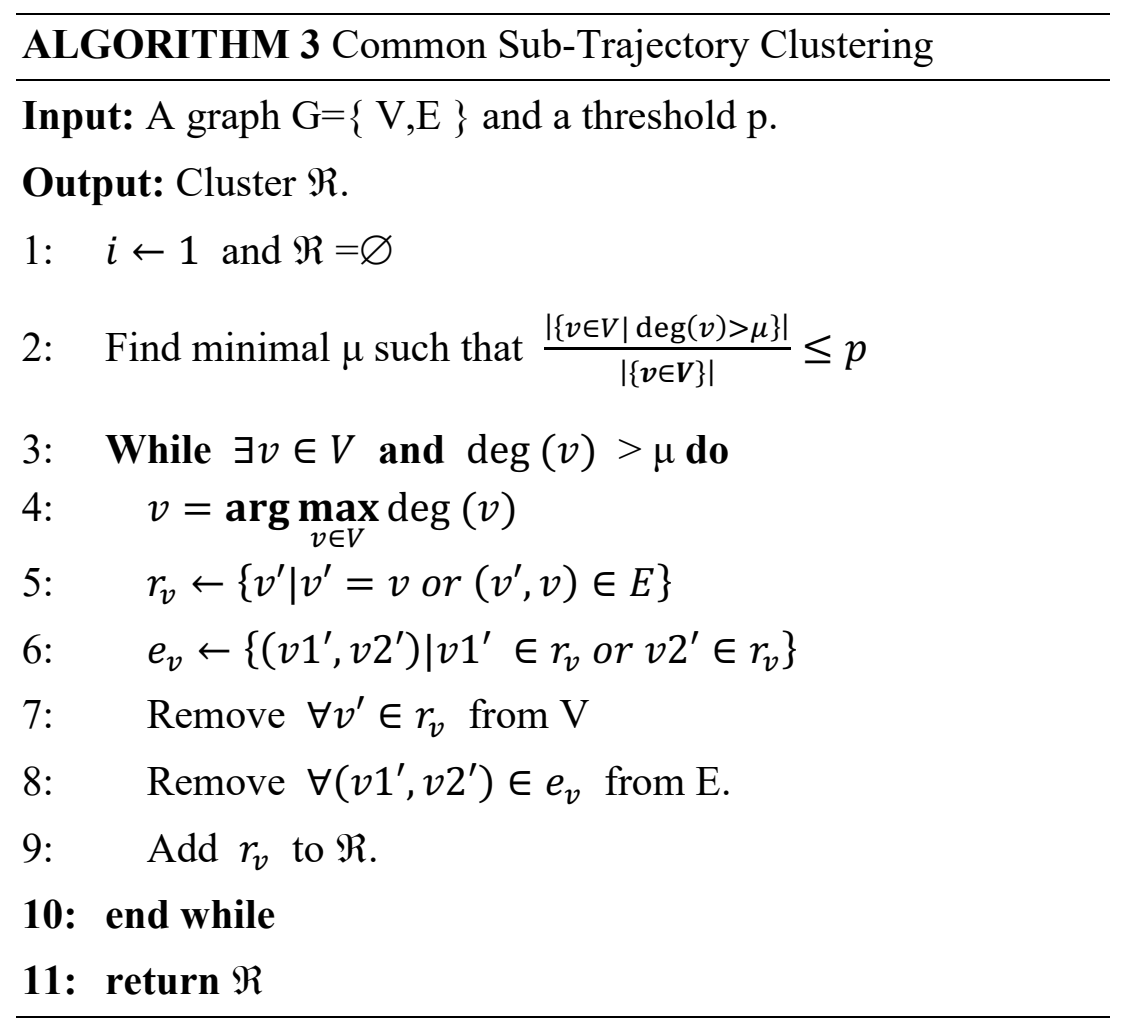

$\ulcorner$ In the input of this stage, clustering requires $\boldsymbol{G}$, information related to the intersection of Hypercubes, and threshold $p . \boldsymbol{G}$ is merged from the results of ALGORITHM 3 using every pair of Hypercube sequences.

One of the important parameters is threshold $p$, which represents a ratio the value of which can be any decimal between 0 and $1 . p$ is the normalization of $\mu$, which represents the minimal acceptable degree of $v$, where $v$ is a Hypercube. $\operatorname{deg}(v)$ represents the degree of $v$, which is the number of trajectories sharing any Hypercubes intersection 
with $v$. Essentially if $p$ is 0.1 , then only the $10 \%$ with the highest degree of $\boldsymbol{V}$ are considered.

The proposed algorithm is outlined in the following. The clustering algorithm first finds $\mu$ based on $p$. The algorithm then finds the vertex with the highest degree that is larger than $\mu$ in $\boldsymbol{G}$. The algorithm then clusters $v_{i}$ and all of the vertices connected to $v_{i}$. The longitude, latitude, time, and direction of all vertices in this cluster are averaged. The vertices and relative edges in the cluster are then removed from $\boldsymbol{G}$. This process of finding-clustering-removing is repeated until the degree of each vertex is smaller than $\mu$, or no vertices remain in $\boldsymbol{V}$. Any vertices that do remain in $\boldsymbol{V}$ are treated as outliers and disregarded. The output of the algorithm is a set of clusters, each of which has four attributes: longitude, latitude, time, and direction. The output examples are shown in “Applications", section 6. $\lrcorner[4]$

$$
\begin{gathered}
r . x=\frac{\sum_{c_{i} \in r} c_{i} \cdot x}{\left|c_{i} \in r\right|}, r \cdot y=\frac{\sum_{c_{i} \in r} c_{i} \cdot y}{\left|c_{i} \in r\right|}, r . t=\frac{\sum_{c_{i} \in r} c_{i} \cdot t}{\left|c_{i} \in r\right|} \\
r . \theta=\arctan \left(\frac{\sum_{c_{i} \in r} \sin \left(c_{i} \cdot \theta\right)}{\sum_{c_{i} \in r} \cos \left(c_{i} \cdot \theta\right)}\right)
\end{gathered}
$$

The centroid of each $\mathrm{r}$ is the average of all member centers, as (8). Calculating the average direction is more complicated than determining longitude, latitude, and time. This is because the angles cannot be summed directly and divided by the total number. This problem must be solved using (9) 


\subsection{Parameter Determination}

This study designed ALGORITHM 4 to automatically find the parameters for the preprocessing and ETA prediction steps. Users can input a set of latitude sequences, longitude sequences, and time to obtain $d_{x}, d_{y}$, and $\tau$, respectively. $\gamma$ and $\mathrm{m}$ are metaparameters. The default values of $\gamma$ and $m$ were 0.8 and 5, respectively, representing $80 \%$ of hypercubes that involve at least five points.

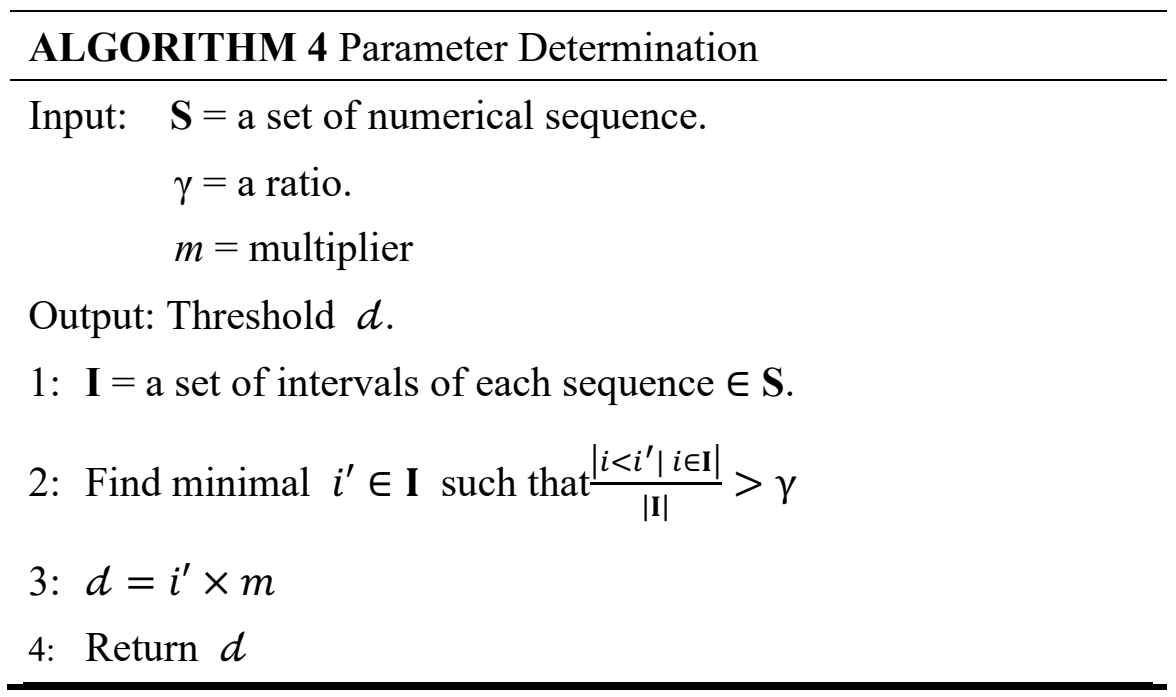




\subsection{HyperETA Algorithm}

This section describes the architecture of HyperETA. FIGURE 12 presents a schematic of the architecture, including the training process and the prediction process. In the training process, the trajectory model and parameters are determined. In the prediction process, the ETA of a given trajectory is predicted. The dotted lines represent that the connected components are identical.

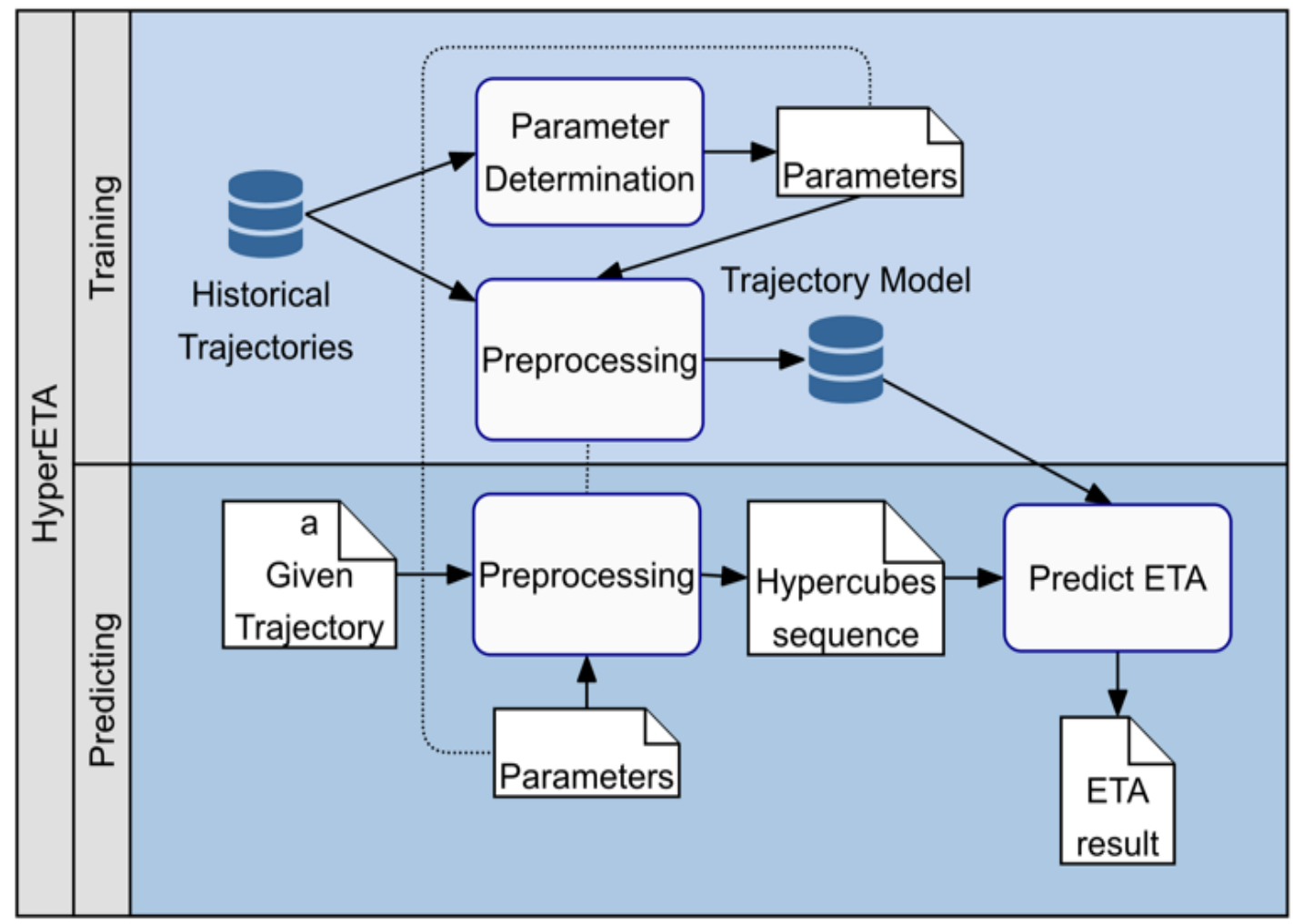

FIGURE 12. HyperETA architecture. 
HyperETA is based on Hypercube clustering. ALGORITHM 5 is the detail of predicting ETA. First, the input is a given hypercube series and a trajectory model (TM). The model includes a hypercube series, original trajectories, and a mapping table that maps the hypercubes to the original trajectories. The trajectory model was produced by storing each result of ALGORITHM 1 that transformed each historical trajectory into Hypercube series. The remaining inputs $d_{x}, d_{y}$, and $\tau$ are determined by ALGORITHM 4. The input $\theta$ is the angle difference between two hypercubes that can be tolerated by Hypercube_Intersection(). The output is the predicted ETA of Cgiven.

Lines 2-6 predict the ETA of each given Hypercube. In Line 3, the Hypercube_Intersection() function is ALGORITHM 2. Line 4 further apply DTW [41] to find the most similar subtrajectory in the set $\mathrm{C} \cap$ to increase accuracy. Min_DTW_Cube() also compare the GPS points amount between Hypercubes; it will be less similar if a difference of the amount in a compared hypercube is less than half of the amount in " $c$ ". The next GPS point on the subtrajectory is counted as well. The time cost of the subtrajectory is accumulated into TotalTime.

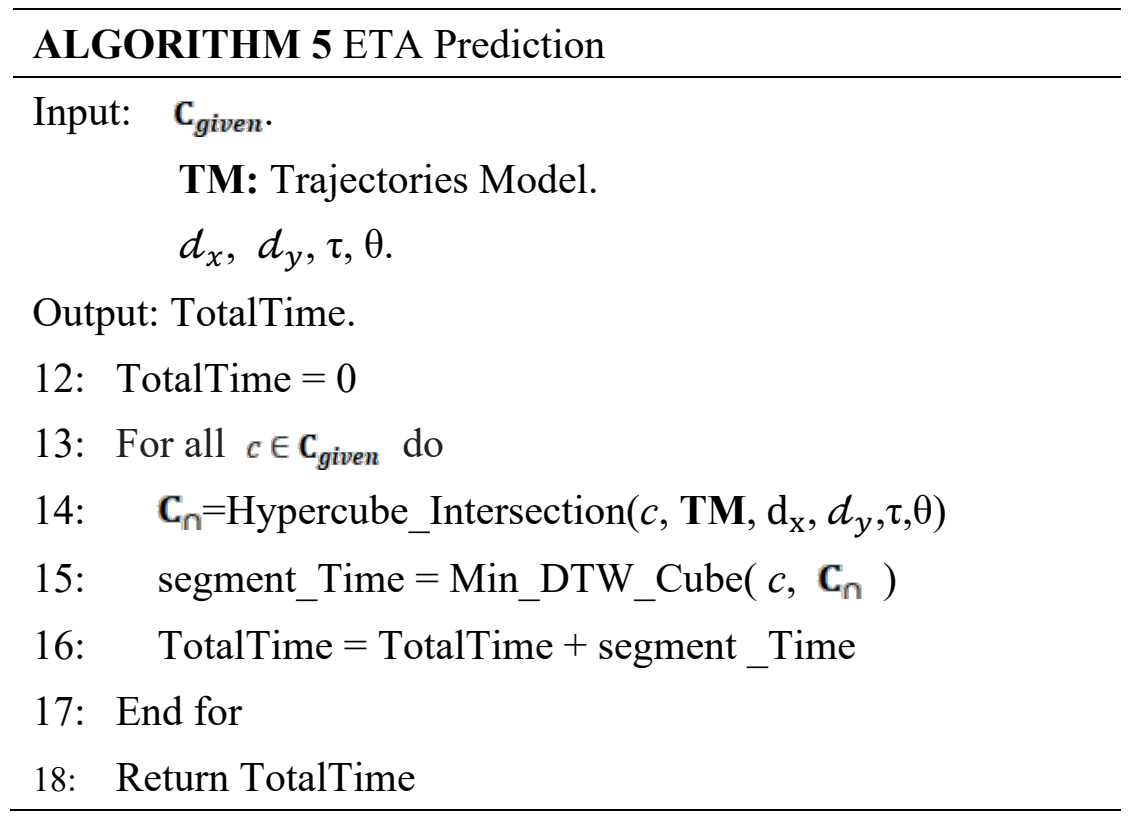




\subsection{The optimization}

One of the definitions of clustering is that "Minimizing total variation of a group; maximizing total variation between groups." I tried to adapt that characteristic to my method. I test it on a small part at first.

Back to the Algorithm 3 Line $4\left\ulcorner v=\arg \max _{v \in V} \operatorname{deg}(v)\right\lrcorner$, the original design is that if there are many $\mathrm{v}$ that fit the condition, the first one will be taken for the following process. Therefore, I test the definition by recursively find the best first one, as algorithm $6 . \operatorname{deg}(v)$ represents the degree of $v$, which is the number of trajectories sharing any Hypercubes intersection with $v$, as the definition in section 4.3. Despite the good or the bad effects of the best first one( It needs completely experiments and it will become a new study), this method significantly slow down the processing speed of "Common sub-trajectories clustering" and is impractical to use. Because the modification is just to decide the best first one, the meaning is not important. Also, it exceeds my research domain. Algorithm 6 is not in the main contents.

Furthermore, if I totally adapt the technique in Algorithm 6 to Algorithm 3 to find theoretically the best clusters instead of my original greedy technique, despite it will make the performance of my method too slow to be used, this technique may involve an area that is too huge( Spreading out from the center of a cluster, like a chain reaction, there are too many hypercubes that have intersections indirectly). The results will be like the test results of DBSCAN, it became one huge block(cluster) and it's meaningless to my application. It exceeds my research domain. 


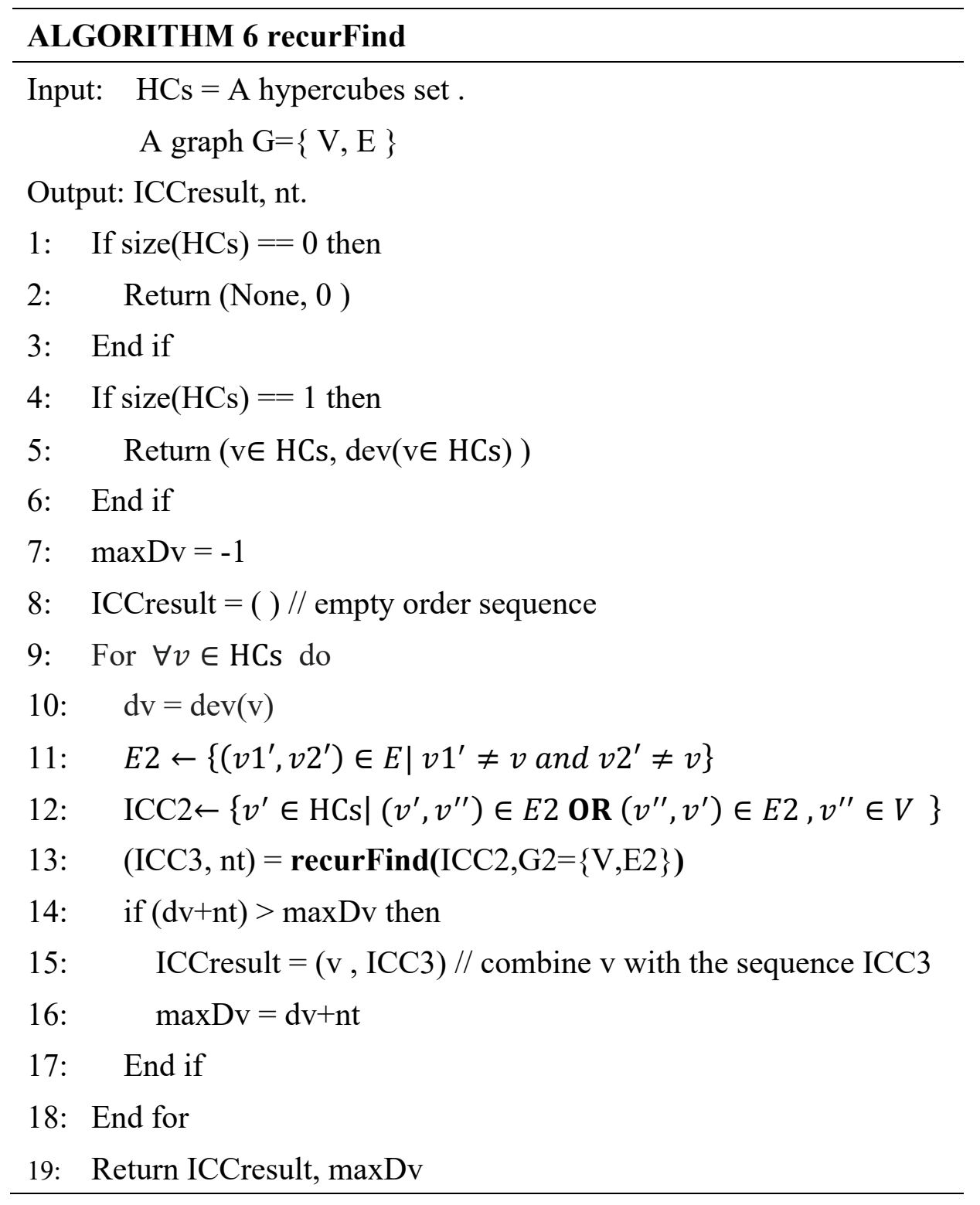




\section{EXPERIMENTS}

Our objective in section 5.1 was $\ulcorner$ to determine whether the definitions of common sub-trajectories (1), (2) and (3) are satisfied. Section 5.2 compares Hypercube clustering with grid clustering in terms of effectiveness and performance. Section 5.3 was meant to compare the accuracy of the clustering results obtained using Hypercube clustering, grid clustering, and TraClus.

The dataset ${ }^{1}$ contains 17,621 GPS trajectories with a total distance of $1,292,951$ kilometers and a total duration of 50,176 hours by 182 users. Those trajectories were recorded by various GPS loggers and GPS-phones with various sampling rates. $91.5 \%$ of the trajectories are logged in a dense sampling rate, e.g., every $1 \sim 5$ seconds or every $5 \sim 10$ meters per GPS point. Each record in the dataset contains the information of latitude, longitude, altitude, and timestamp. This dataset recorded a broad range of user movements, including everyday routines like going home, going to work, and some entertainments and sport activities such as shopping, sightseeing, dining, hiking, and cycling. To reduce variation, This study only uses the GPS points in Beijing city inside 6th Ring Rd.

The parameters ( $\boldsymbol{d}=100$ meters, $\tau=3600$ seconds, or smaller values) will be used in the following experiments. The values are from the previous work [5] while this study assumes the maximal spatial gap between two paths where can share a ride is 100 meters, and the maximal time difference is assumed 3600 seconds to represent the maximal waiting time that the early one can wait. $\lrcorner[4]$

\footnotetext{
${ }^{1}$ https://www.microsoft.com/en-us/download/details.aspx?id=52367
} 
Lab Environment The experiments were carried out under the following specifications:

- CPU: Intel Core i7-4790 3.6GHz

- Memory: DDR3-1600 16GB Non-ECC

- Storage: Intel SSD 535 series $240 \mathrm{~GB}$

Section 5.1,5.2,5.3

- OS: Windows 7 64bits

- Program Language: C++ on Visual Studio 2013

Section 5.4

- OS Ubuntu Linux 19.10 64-bit

- Program Language: Python 3.7.7 (HyperETA)

- Python 2.7 + PyTorch 1.4 + CUDA 10.1 + cudnn 8.0.3(DeepTTE)

- GPU GEFORCE GTX750Ti-DC2OC-4GD5

$\left\ulcorner\right.$ The programming language used for TraClus $^{2}$ is Python, which differs significantly from $\mathrm{C}++$. This makes it very difficult to compare TraClus with Hypercubes and Grid clustering in terms of efficiency. This study had tried to do the same experiment, section 5.2, on TraClus. After the software loaded the 1GB dataset, all of the computer memory was occupied. The software was crashed before getting a result. It is shown that the entire Geolife dataset is too big for the software. Thus, the time cost and memory usage of TraClus are not included in the comparison in section 5.2. $\lrcorner[4]$

\footnotetext{
${ }^{2}$ https://github.com/apolcyn/traclus_impl
} 


\subsection{Validation of a Hypercube}

$\ulcorner$ This experiment was meant to validate the correctness of the Hypercube; i.e., whether the definitions of common sub-trajectories, (1), (2), and (3), are satisfied. Theoretically, the maximal geographic distance between two points in two Hypercubes with a common intersection is as follows:

$$
\sqrt{\left(\frac{3}{2} d_{x}\right)^{2}+\left(\frac{3}{2} d_{y}\right)^{2}}
$$

To simplify the experiments, let $d_{x}=d_{y}=\boldsymbol{d}$, and use two sets of values ( $\boldsymbol{d}$ $=20$ meters, $\tau=600$ seconds $)$ and $(\boldsymbol{d}=100$ meters,$\tau=3600$ seconds $)$. After Hypercube intersection, Dynamic Time Warping (DTW) [20] [21] was used to compute the pairpoint distance between sub-trajectories in Hypercubes with an intersection relation.

FIGURE 13 presents the histogram for $\boldsymbol{d}=20$ and $\tau=600$; there is $92 \%$ of the pair-points separated by less than $\boldsymbol{d}=20$ meters. FIGURE 14 presents the histogram for $\boldsymbol{d}=100$ and $\tau=3600$; there is $95 \%$ of the pair-points separated by less than $\boldsymbol{d}=100$ meters. Our results show that the distance of most of the common sub-trajectories was less than $d$. In general, it can be assumed that $\varepsilon$ represents the maximal geographic distance in a common sub-trajectory. $\lrcorner[4]$ 


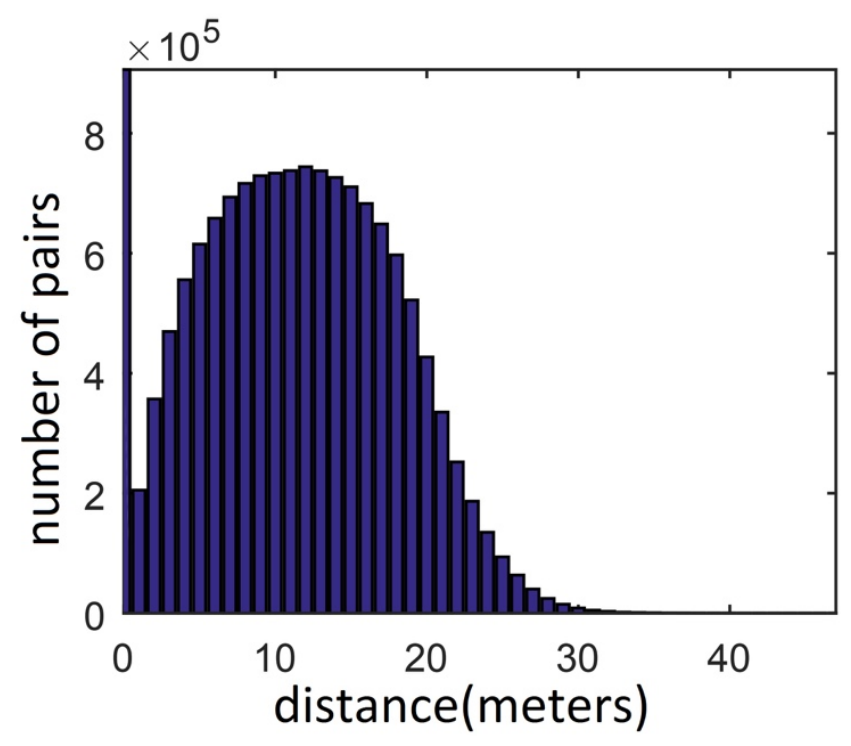

FIGURE 13. Accumulation results of hypercube size $\boldsymbol{d}=20$ and $\tau=600$.

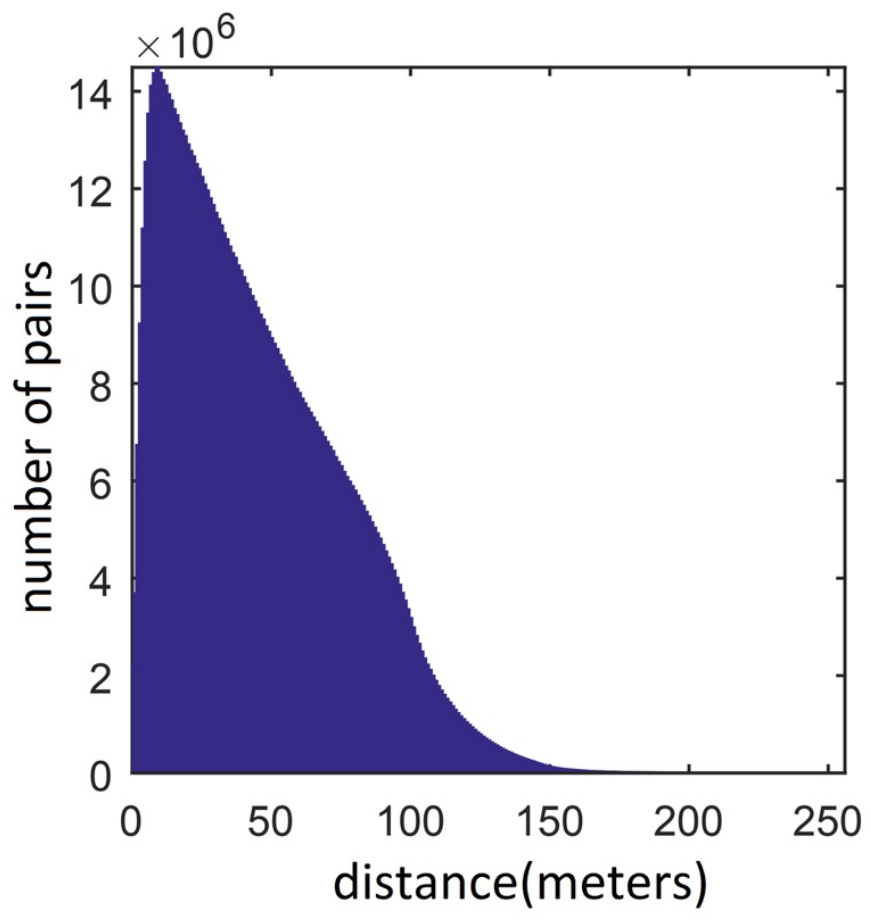

FIGURE 14. Accumulation results of hypercube size $\boldsymbol{d}=100$ and $\tau=3600$. 


\subsection{Hypercube Clustering vs. Grid Clustering}

$\ulcorner$ This study compared Hypercube clustering with grid clustering in terms of efficacy and performance. Nine test cases were generated using the following combination of parameters: $\boldsymbol{d}=24$ meters, 50 meters, or 100 meters; and $\tau=10$ minutes, 30 minutes, and 1 hour. The Geolife dataset is used as testing data. $\lrcorner$ [4]

\subsubsection{Experimental Design}

$\ulcorner$ The experiment process of Hypercube clustering is described as follows. Nine combinations of $\varepsilon$ and $\tau$ were used in each experiment, and this experiment is performed nine times in other words. After preprocessing, every possible combination of paired trajectories was input into the Hypercube Intersection algorithm. The common subtrajectory distance based on intersection information from the Hypercube intersection is summed up as a result.

This study removed the direction constraint from Hypercube clustering in this experiment to ensure a fair comparison, as the major difference between Hypercube clustering and grid clustering is geographic and temporal space.

The experiment process of grid clustering is as follows. The first trajectory in the dataset was set into the grid. The positions in the grid corresponding to each trajectory in the dataset were then checked to determine whether the grid was occupied by the first trajectory. The distance of the sub-trajectory is accumulated if the block in the grid was occupied. After the checking process, the grid space was initiated. The first trajectory was removed from the dataset. This process was repeated until no trajectories remained in the dataset, whereupon the accumulated distance was compared with the results from Hypercube clustering. $\lrcorner[4]$ 


\subsubsection{Experimental Results}

$\ulcorner$ This experiment evaluated the clustering methods according to three metrics: computation time, memory overhead, and accuracy. Our results show that Hypercube clustering was able to accurately retrieve common sub-trajectories with less computation time and less memory usage.

FIGURE 15 presents the common sub-trajectory distance of the two clustering methods. As can be seen, even when Hypercube clustering was implemented using the smallest combination of parameters, the resulting common sub-trajectory distance was longer than that of grid clustering. $\lrcorner[4]$

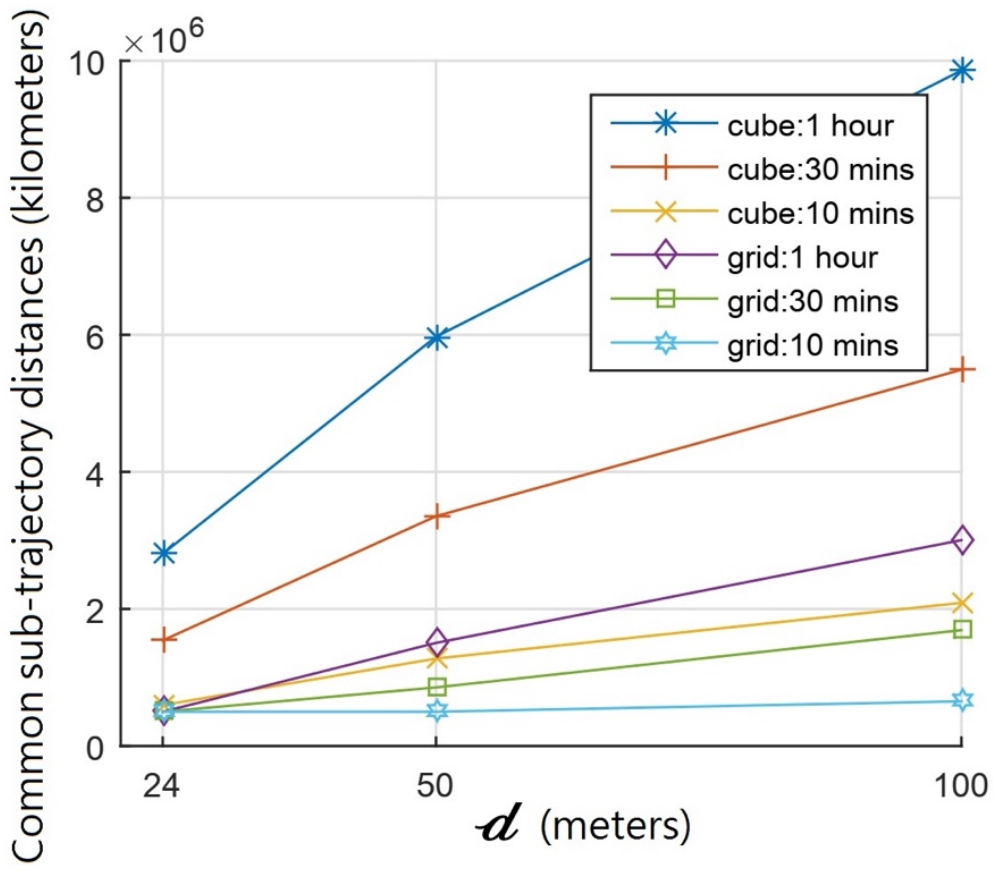

FIGURE 15. Common sub-trajectory distances by clustering under various parameter combinations. 
Grid clustering was outperformed in terms of finding long common subtrajectories because it considers only common sub- trajectories in the same block; i.e., sub-trajectories in adjacent blocks are not considered even when there is only a small gap between them, as shown in FIGURE 16. The same situation also occurs in the temporal space. Hypercube clustering was able to find every common sub-trajectory in cases where the gap between two trajectories is less than $\varepsilon$, as shown in FIGURE 17, and $\tau$ for temporal space.

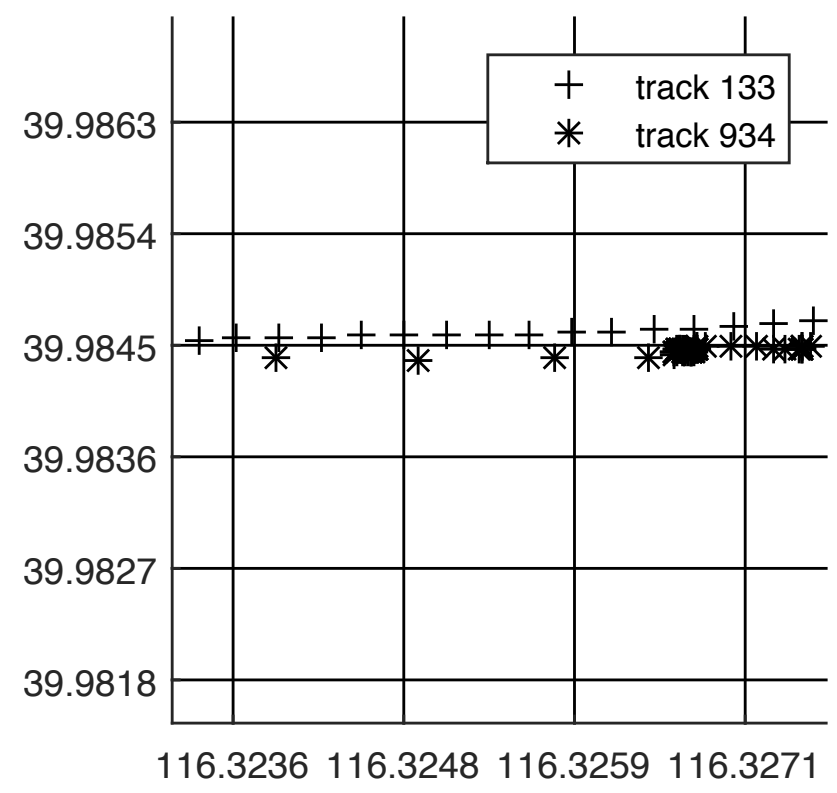

FIGURE 16. An example that illustrates the drawback of grid clustering. Trajectories with short gaps lie in different blocks are overlooked. 


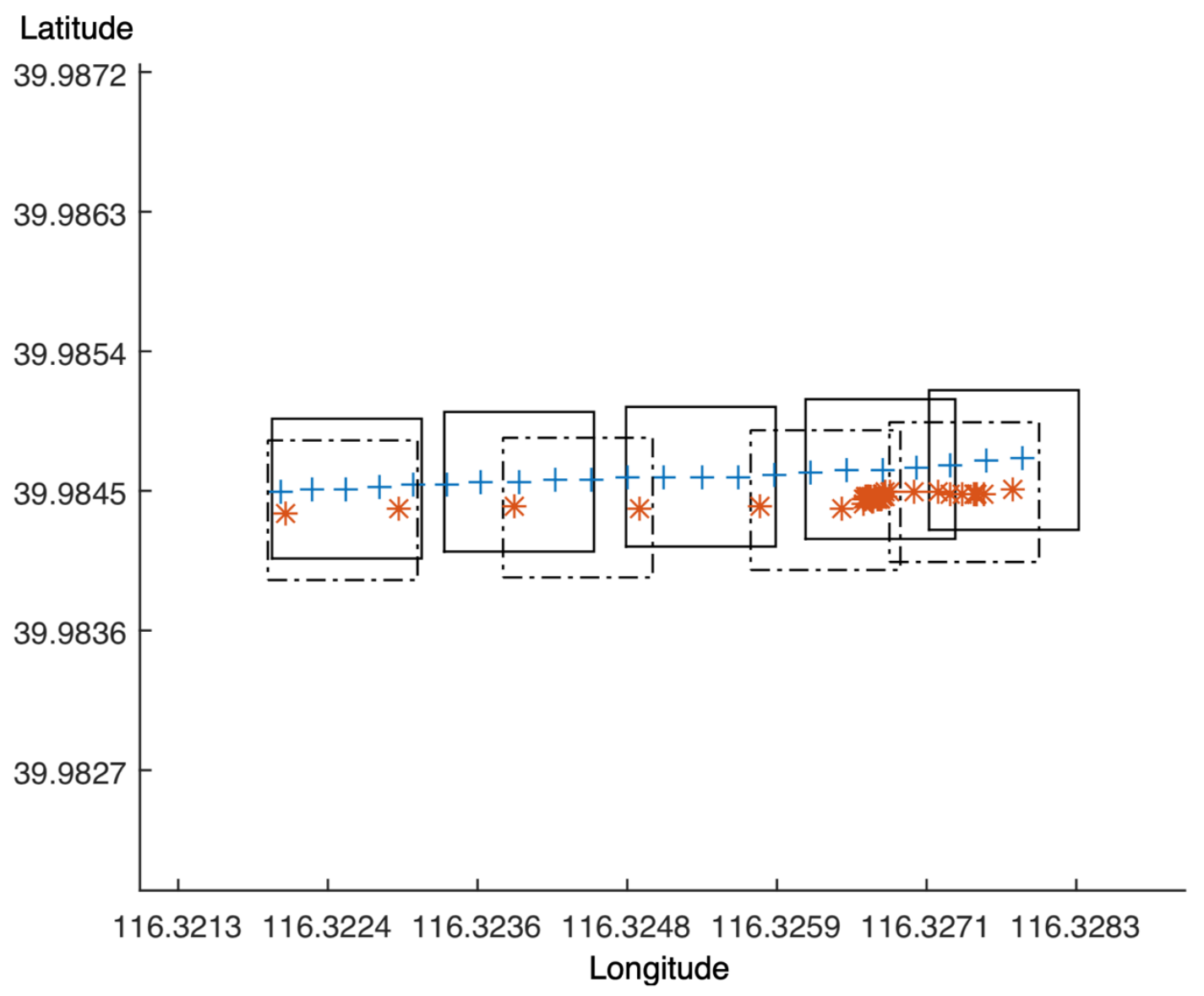

FIGURE 17. The example of Hypercubes Clustering. Trajectories have a very short gap, and the cubes are overlapped each other. The dot dashed lines are the cube range of red star symbol “*”. 
$\ulcorner$ One of the advantages of Hypercube clustering is that a reduction in temporal size is reflected in a reduction of computation time, as shown in FIGURE 18. This is to be expected because a larger temporal size should provide a larger number of common sub-trajectories requiring computation. The situation is the opposite of grid clustering, which requires more computation time for grids of smaller size, as shown in FIGURE 19.

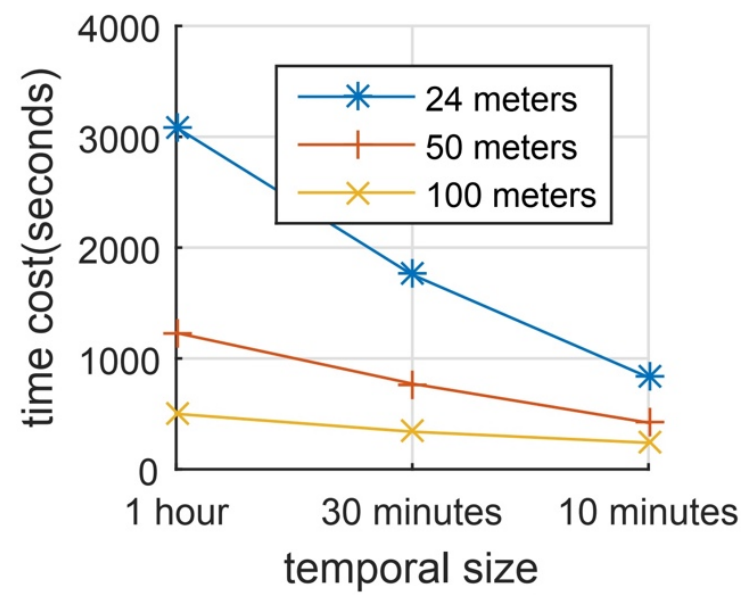

FIGURE 18. Computation time required for Hypercube clustering.

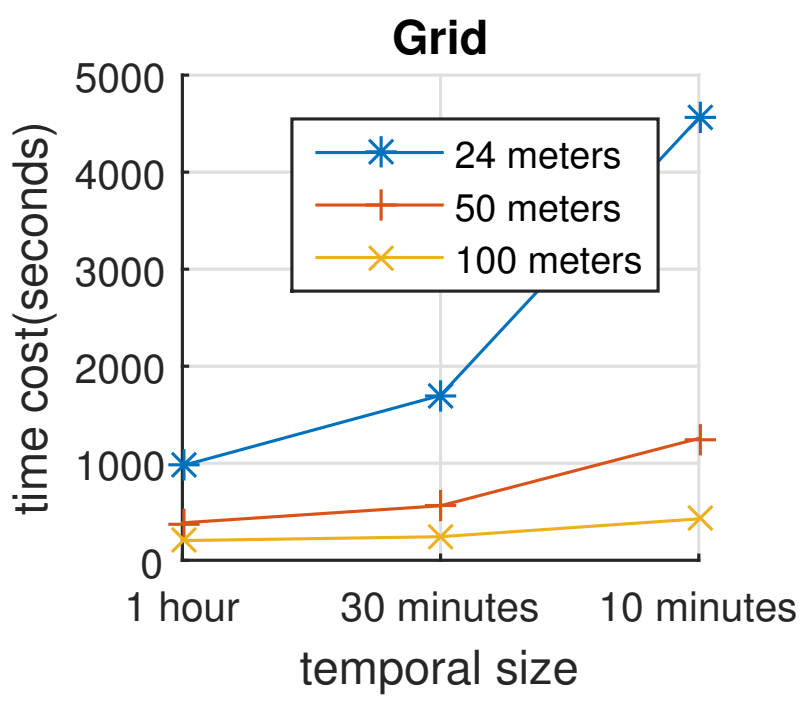

FIGURE 19. Computation time required for grid clustering. 
FIGURE 20 presents a number of comparisons used to check the intersections created by Hypercube clustering under various temporal sizes. Our results show that the number of comparisons decreased with temporal size. As mentioned in Section 4, the algorithm first checks the maximal time and minimal time of two Hypercube sequences. A smaller temporal size implies fewer Hypercube intersections in the time domain, thereby eliminating unnecessary checking. For each Hypercube, a reduction in the temporal size reduces the number of intersecting Hypercubes that must be checked.

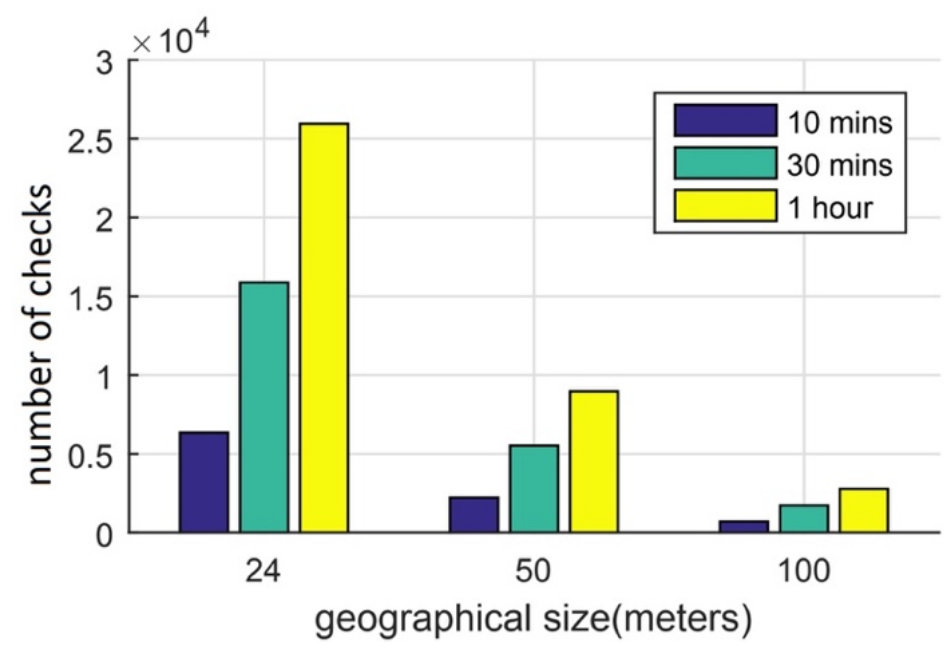

FIGURE 20. Hypercubes: number of intersections to be checked.

The computation time of grid clustering revealed the opposite results. The smaller temporal size was shown to increase computation time. As shown in FIGURE 19, the computational time of the case where $d=24$ meters and $\tau=600$ seconds was 4.5 times longer than the where case $\boldsymbol{d}=24$ meters and $\tau=3600$ seconds. There are two reasons for this. First, the number of grid cells containing trajectories increases with a reduction in temporal size, thereby necessitating more computation. The second reason is that grid clustering requires to initialize the contents of grid cells which represented by a huge array. When the temporal size is smaller, the size of the array is larger, such that the time to initialize is long. 


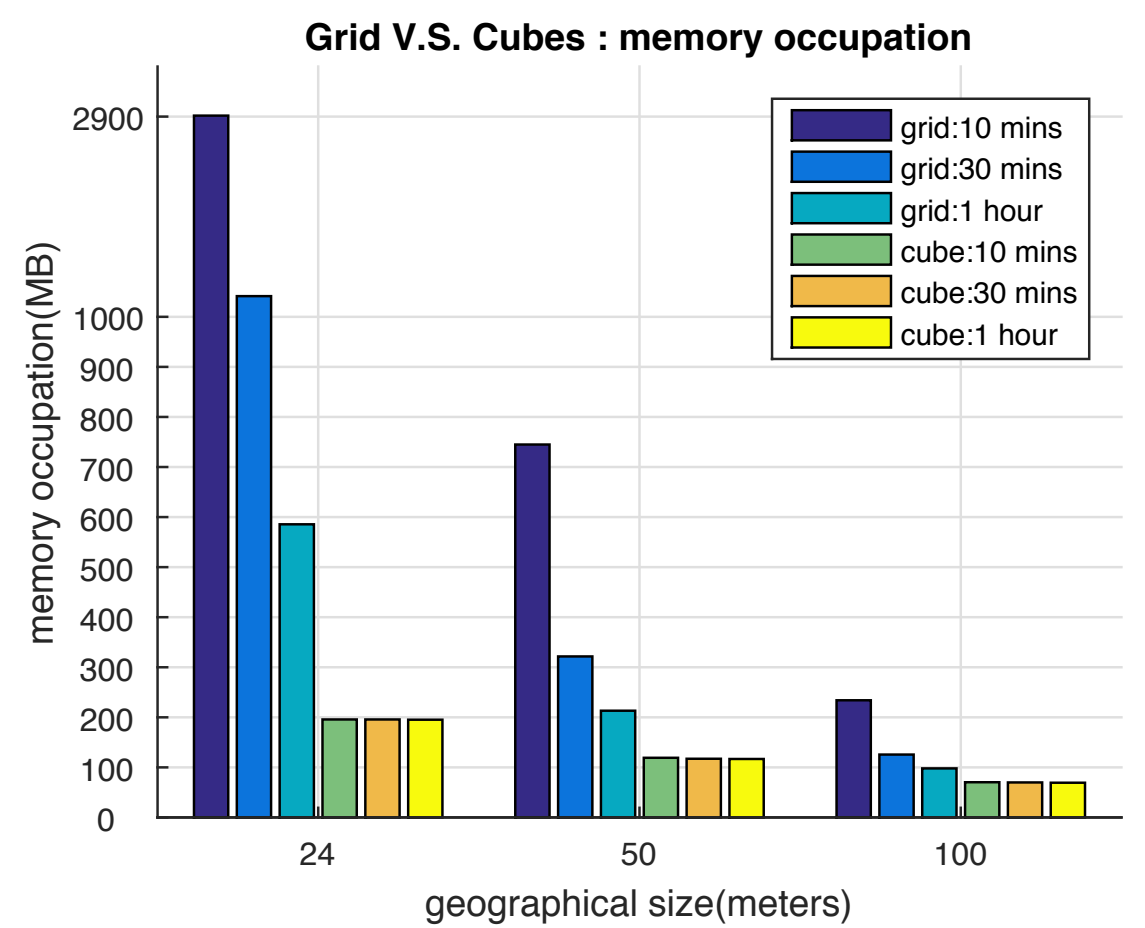

FIGURE 21. Grid vs. Hypercubes: memory usage. "cube”' represents Hypercube.

The total memory consumption of Hypercube clustering is significantly less than that of grid clustering. As shown in FIGURE 21, Hypercube clustering requires less memory than does grid clustering. Furthermore, the memory usage of Hypercube clustering remains similar, regardless of the parameter combination. $\lrcorner[4]$

\subsection{Accuracy of Clustering}

$\ulcorner$ The accuracy of Hypercube clustering was evaluated using Qmeasure. The test data included the number of trajectories generated from duplicated real trajectories. Note that each duplication was randomly shifted in the spatiotemporal space. The duplications were clustered using Hypercube, Grid, and TraClus to derive the real trajectory from the duplications.

The formula used for Qmeasure[1] was (10) where $T$ is a set of all real trajectories, $p$ is a GPS point in $t, C_{t}$ is the set of clusters generated according to the real trajectory duplications, $\operatorname{dist}(p, c)$ is the point to line distance between a GPS point and a 
cluster. The transformation of each cluster into a line is based on its range and direction $\lrcorner$ [4]. A total of 223,566 trajectory points generated 223,566 different deviations.

$$
\text { Qmeasure }=\frac{1}{|t| \times|T|} \sum_{t \in T} \sum_{p \in t} \arg \min _{c \in C_{t}} \operatorname{dist}(p, c)
$$

The real trajectories in this experiment were those associated with User 2 in Geolife which is also used for the "Popular route" in the Application, section 6.1. Processing all of the trajectories resulted in 223,566 deviations from which to derive statistical results. $\ulcorner$ The number of duplicates varied according to the value of $\boldsymbol{d}$, as follows: 100 duplicates for $\boldsymbol{d}=100$ meters, 25 for $\boldsymbol{d}=50$, and 10 for $\boldsymbol{d}=25$. The temporal parameter $\tau$ is not presented where the results showed no significant differences under three values of the parameter ( 1 hour, 30 minutes, and 10 minutes). 
The experiment results are presented in FIGURE 22. The Hypercube method achieved the smallest Qmeasure in each $\boldsymbol{d}$, indicating the highest accuracy. Even with an increase in the value of $\boldsymbol{d}$, the accuracy of Hypercube remained high. All results obtained using Hypercube were less than 1, which is too small to be shown in the figure. $\lrcorner$ [4]

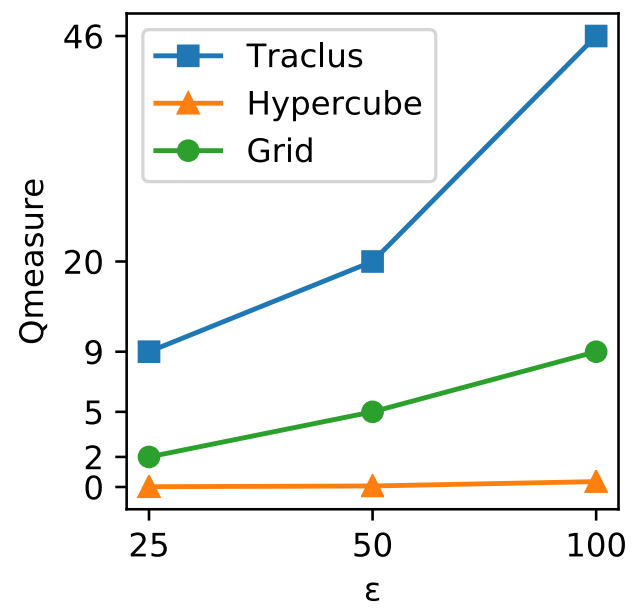

FIGURE 22. Qmeasure of Hypercube, Grid, TraClus clustering.

FIGURE 23, FIGURE 24, and FIGURE 25 illustrate the distribution of deviations in the value of $\boldsymbol{d}$. The percentage refers to the ratio of the number of deviations in

$$
x \leq \text { deviation }<\mathrm{x}+1
$$

to the total number of deviations. Some scales between maximal and minimal scale are disregarded to simplify the figures. 


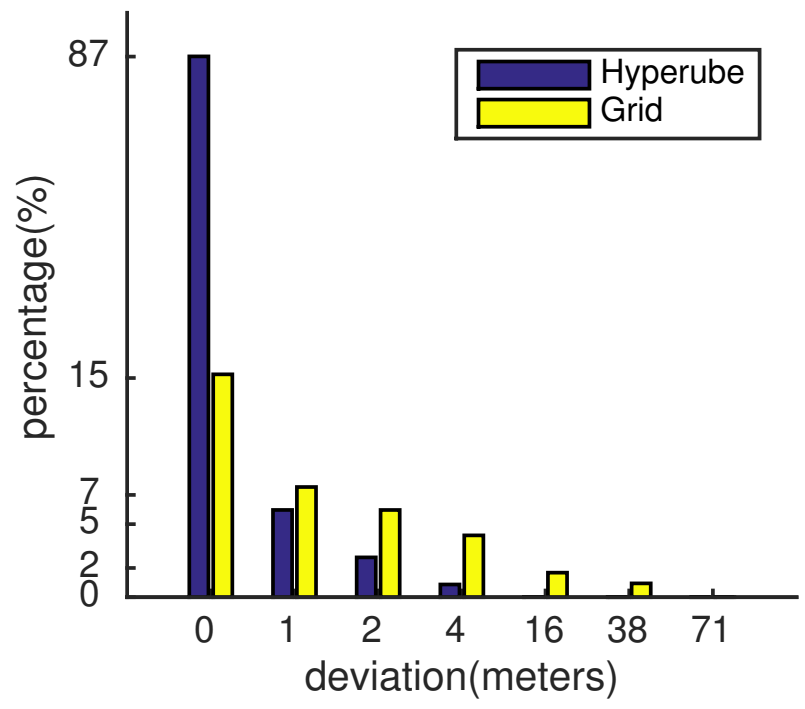

FIGURE 23. Deviations in hypercube clustering and grid clustering using geographic size of $100 \times 100 \mathrm{~m}$

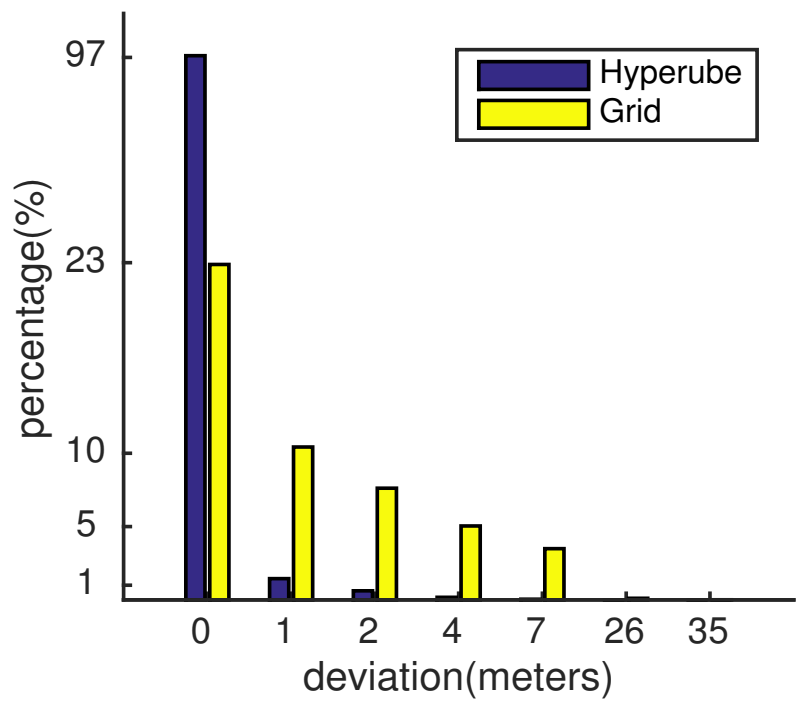

FIGURE 24. Deviations in hypercube clustering and grid clustering using geographic size of $50 \times 50 \mathrm{~m}$ 


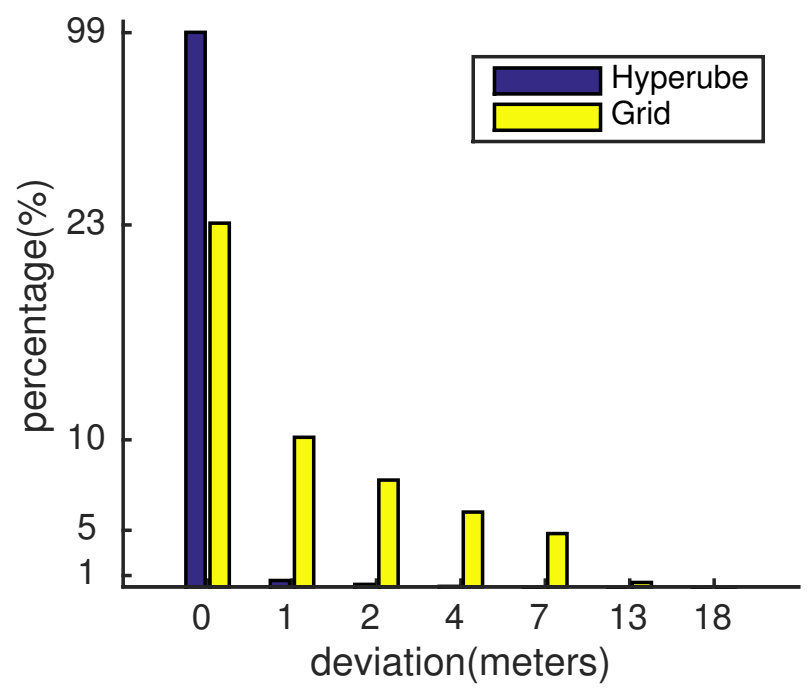

FIGURE 25. Deviations in hypercube clustering and grid clustering using geographic size of $25 \times 25 \mathrm{~m}$

The temporal size is not presented because the results are a sum of three sizes ( 1 hour, 30 minutes, and 10 minutes), and because there were no significant differences in the experiment results obtained using the three sizes. Each figure presents analysis of $223566 \times 3$ deviations. The difference in temporal size was hidden to clarify the figure. 
TABLE 2 presents a comparison of different combinations of parameters and methods. The comparisons include the percentages when $\mathrm{x}=0$ (bigger-the-better) and the maximal deviation on the X-axis (smaller-the-better). Our results show that the Hypercube outperformed the grid in every case, regardless of the value of $\boldsymbol{d}$.

TABLE 2. Comparison of Hypercube and grid methods

\begin{tabular}{c|c|c|c}
\hline $\boldsymbol{d}$ & method & $\mathrm{y}(\mathrm{x}=0)$ & $\max (\mathrm{x})$ \\
\hline \hline \multirow{2}{*}{100} & Hypercube & $87 \%$ & 38 \\
\cline { 2 - 4 } & Grid & $15 \%$ & 71 \\
\hline \multirow{2}{*}{50} & Hypercube & $97 \%$ & 26 \\
\cline { 2 - 4 } & Grid & $23 \%$ & 35 \\
\hline \multirow{2}{*}{25} & Hypercube & $99 \%$ & 13 \\
\cline { 2 - 4 } & Grid & $23 \%$ & 18 \\
\hline
\end{tabular}

\subsection{Accuracy of HyperETA}

\subsubsection{Experimental Design}

Data Description This study evaluated the proposed method on a dataset of taxi trajectories in Cheng-du[7] . This dataset was determined to contain 19,400 trajectories (712000 GPS records) of 4565 taxi drivers in August 2014 in Cheng-du, China. The shortest trajectory contained only 15 GPS records $(2.5 \mathrm{~km})$, and the longest trajectory contained 119 GPS records $(40 \mathrm{~km})$. Moreover, This study found a few abnormal GPS subtrajectories in the training and testing data during the experiments. These abnormal data were removed from the test data in the experiments.

This study compared the following methods in our experiments:

1) DeepTTE, which uses a deep learning framework, PyTorch, to predict ETA.

2) DeepTTE-GPU, which uses GPUs(graphics processing units) to accelerate the computational speed, which is common in PyTorch. 
3) HyperETA-noDTW, which is the first HyperETA before the application of DTW.

4) HyperETA, which is the primary method developed in this study.

Criteria This study derived mean absolute percentage error (MAPE) values to evaluate the prediction results. MAPE expresses the accuracy as a ratio, as presented in (1):

$$
\mathrm{MAPE}=100 \% \times \frac{1}{n} \sum_{i=1}^{n}\left|\frac{A_{i}-F_{i}}{A_{i}}\right|
$$

where $A_{i}$ is the actual value and $F_{i}$ is the forecast value.

Mean absolute error (MAE) and root mean square error (RMSE) were also used in the experiments. MAE is an arithmetic average of the absolute errors; that is, the average error between the actual value and forecast value. Regarding RMSE, large errors emerge from small errors.

Meta-parameter setting In this experiment, the meta-parameters $\gamma$ and $\mathrm{m}$ were set to 0.8 and 5, respectively, for Algorithm 2 of HyperETA. The setting represents that most of the hypercubes, approximately $80 \%$, would involve at least five GPS points. $\theta$ was set to $10^{\circ}$.

The software used in the experiments has been released on Github, including HyperETA $^{3}$ and DeepTTE 4

\subsubsection{Accuracy Comparison}

3 https://github.com/oscarhsu/HyperETA

${ }^{4}$ https://github.com/oscarhsu/DeepTTE 
The experimental results are listed in TABLE 3 and TABLE 4.

TABLE 3. PERFORMANCE COMPARISON WITH TEST DATA

\begin{tabular}{|l|r|r|r|}
\hline & MAPE $(\%)$ & \multicolumn{1}{c|}{ RMSE } & \multicolumn{1}{c|}{ MAE(sec) } \\
\hline DeepTTE & 37.36 & 695.70 & 553.36 \\
\hline DeepTTE-GPU & 39.12 & 649.88 & 509.30 \\
\hline HyperETA-noDTw & 21.53 & 434.12 & 279.68 \\
\hline HyperETA & $* \mathbf{2 0 . 5 2}$ & $* 419.23$ & $* 269.72$ \\
\hline
\end{tabular}

TABLE 4. TRAINING LOSS

\begin{tabular}{|l|r|r|r|}
\hline & MAPE(\%) & RMSE & MAE(sec) \\
\hline DeepTTE & 16.88 & $* 142.61$ & 91.03 \\
\hline DeepTTE-GPU & 25.75 & 468.41 & 381.49 \\
\hline HyperETA-noDTW & 3.14 & 191.31 & 57.51 \\
\hline HyperETA & $* 3$ & 189.92 & $* 55.25$ \\
\hline
\end{tabular}

Test Data columns present the data obtained by the algorithms. HyperETA significantly outperformed DeepTTE and DeepTTE-GPU on these data. In HyperETA, the MAPE rate was only $20.52 \%$; moreover, this algorithm had the most favorable RMSE and MAE values. HyperETA outperformed HyperETA-noDTW, indicating that HyperETA coupled with the DTW technique increased accuracy. The error rate of the deep-learning-framework-based DeepTTE was 33.63\%. The error rate of DeepTTE-GPU, which uses a GPU instead of a CPU, was higher at 39.12\%.

Training loss columns present the data predicted by each algorithm for model training. Intuitively, the results should be perfect with near-zero error because the data were already learned. However, DeepTTE yielded distinct results, with a significant error rate of $16.88 \%$. For DeepTTE-GPU, the error rate was $25.75 \%$, which was higher than that of the CPU version. HyperETA and HyperETA-noDTW yielded normal results, and their error rates were only $3 \%$ and $3.14 \%$, respectively, which were considerably lower 
than those of DeepTTE and DeepTTE-GPU. The RMSE of HyperETA with training data was slightly higher than that of DeepTTE, but the MAE was smaller, similar to the other experimental values. This indicates that HyperETA exhibited some differentials in predicting long trajectories. However, the differentials occupied only small parts of the long trajectories, explaining why the MAPE of HyperETA was considerably lower than those of the other techniques.

Distance FIGURE 26, FIGURE 27, and FIGURE 28 present a comparison of the model performance levels for trajectories of different lengths. HyperETA outperformed DeepTTE for every path length. As illustrated in FIGURE 26, most of the MAPE values of DeepTTE were higher than 22\%, but those of HyperETA were lower than $16.2 \%$. After 5-10 distances, the MAPEs of HyperETA decreased significantly. As indicated in FIGURE 27, the RMSEs of DeepTTE increased with distance. However, HyperETA exhibited an opposite phenomenon, where the RMSE values decreased. As shown in FIGURE 28, the predicted MAE of DeepTTE (in seconds) increased with distance. However, the prediction quality of HyperETA remained steady, regardless of the distance. For most trajectory lengths, the experimental values yielded by HyperETA were superior to those yielded by HyperETA-noDTW. However, HyperETA-noDTW yielded lower MAPE, RMSE, MAE values for the distances 0-5. 


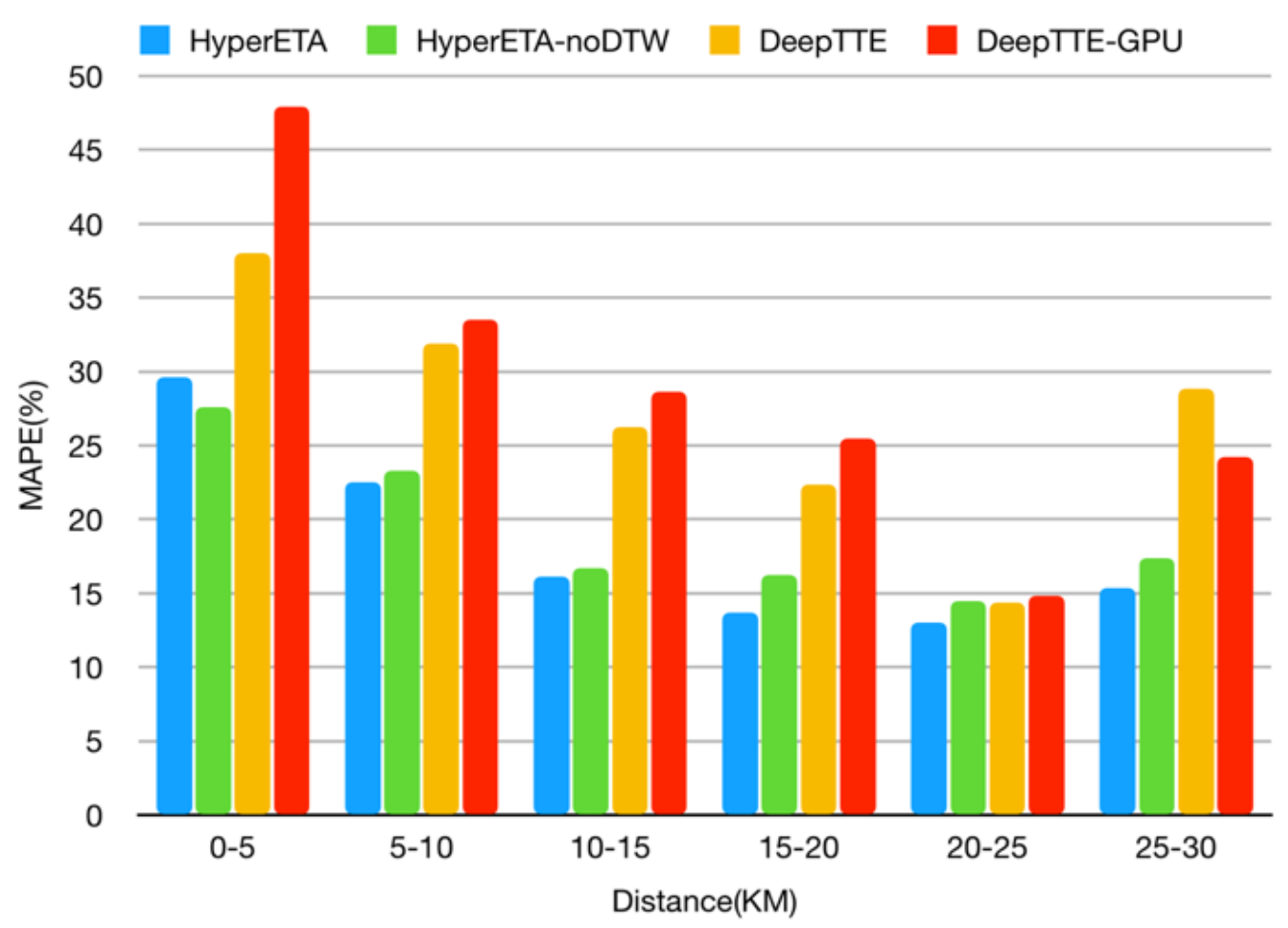

FIGURE 26. Error rates of trajectories having different lengths.

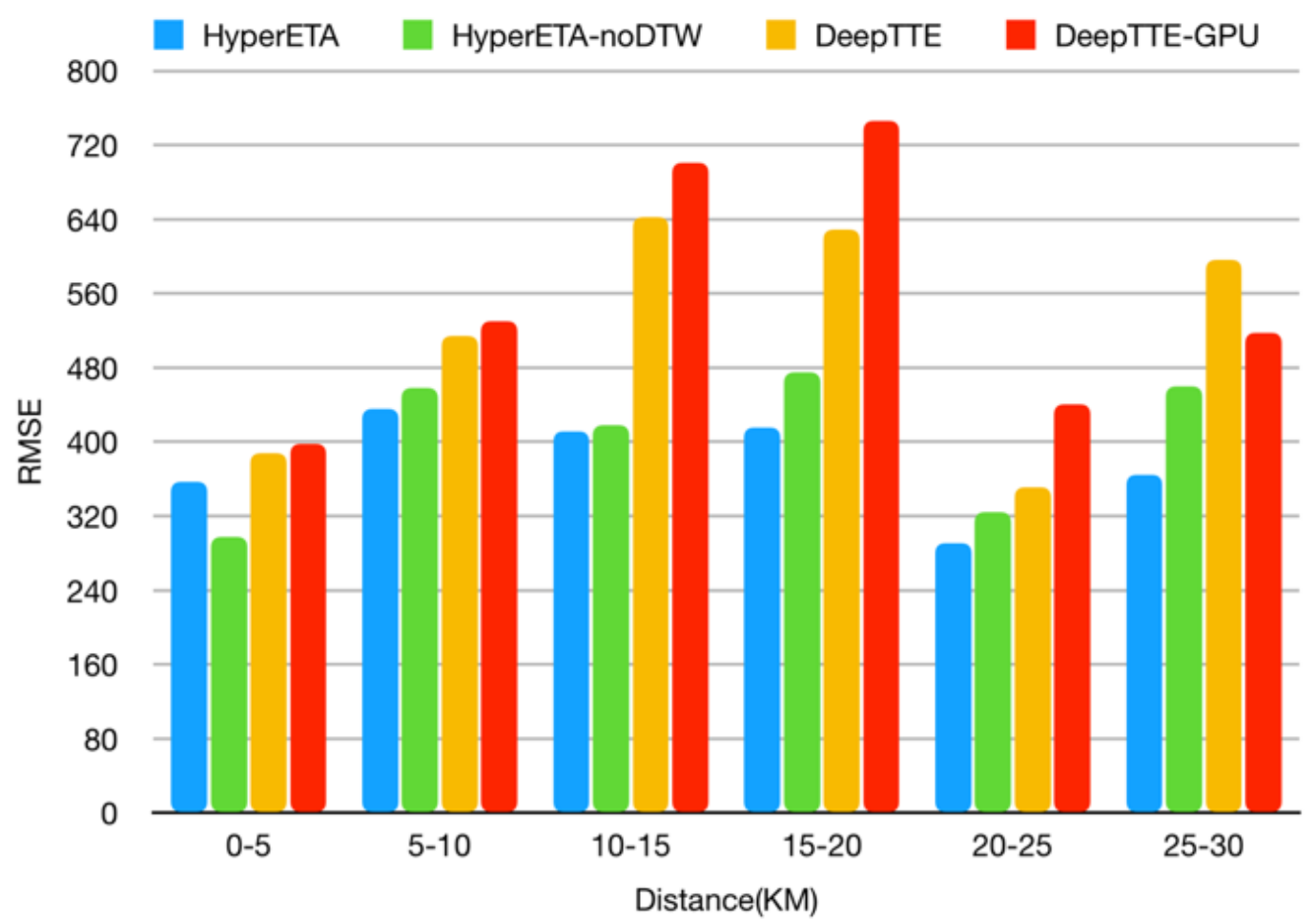

FIGURE 27. RMSEs of trajectories with different lengths. 


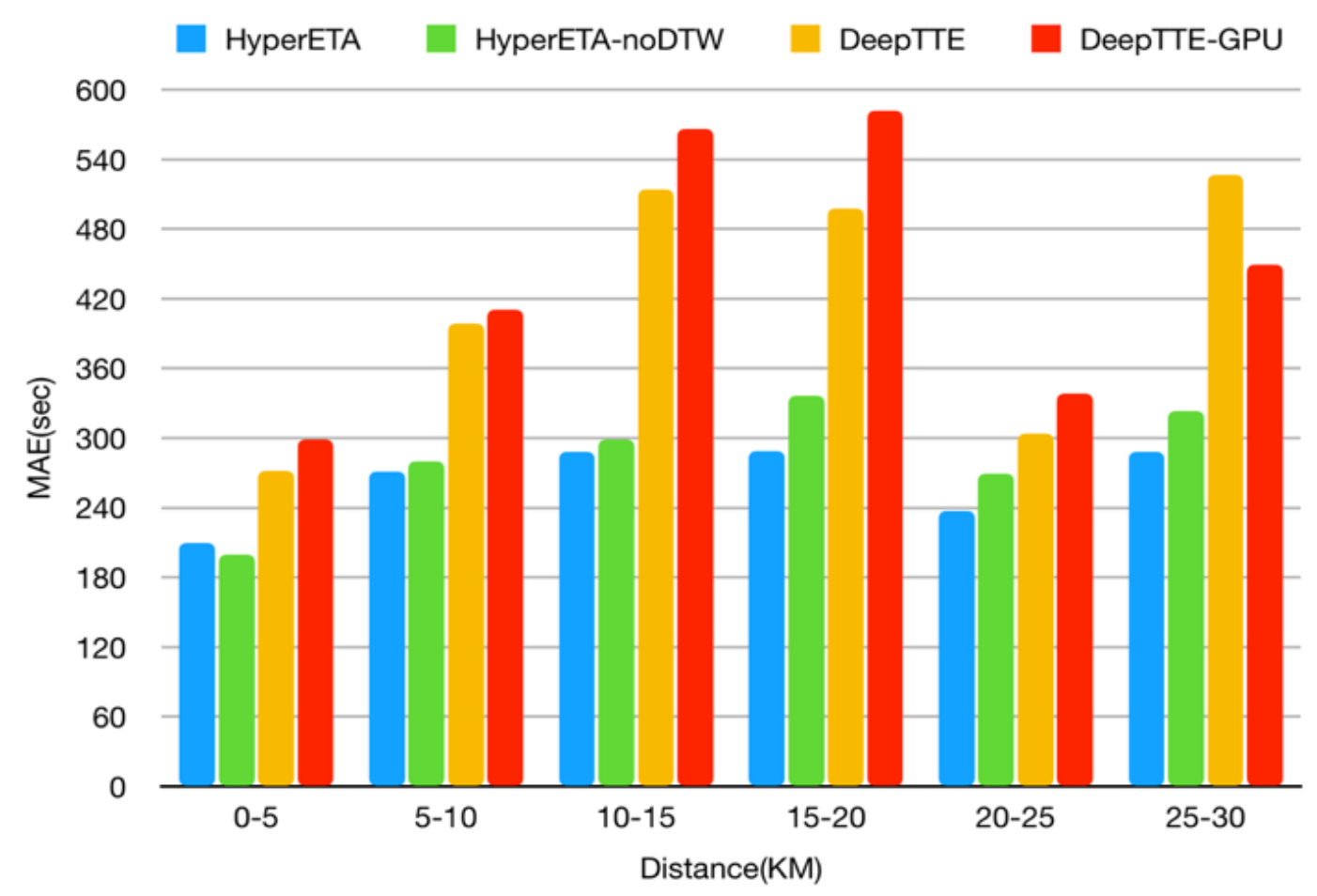

FIGURE 28. MAEs of trajectories with different lengths.

\subsubsection{Discussion}

Data Size HyperETA outperformed DeepTTE. DeepTTE requires 5 million training data items to achieve acceptable accuracy. The training dataset used in this study was only a small portion of the original Cheng-du dataset. Nevertheless, HyperETA offered a higher level of precision than did DeepTTE with the same quantity of training data. This demonstrates that deep learning requires a considerable quantity of labeled data to solve problems; it is impractical if the data volume is limited.

GPU DeepTTE-GPU yielded poorer results than those of DeepTTE because the GPU precision was low. This study used a GTX750Ti, a consumer-level GPU that is inferior to the supercomputing GPU "TITAN V," for example. In general, consumer-level GPUs can only process single-precision floating-point numbers. By contrast, 
supercomputing GPUs can process double-precision floating-point numbers. This considerably influences accuracy because each value in the feature vectors in DeepTTE is normalized to a floating-point number between 0 and 1. Narang[42] described the issue of the single-precision format (FP32) and how it influences gradient descent in backpropagation. PyTorch-1.6 uses a certain method to increase computational speed, which allows it to have the speed of FP16 while maintaining the accuracy of FP32. The default precision of a CPU is FP64 (i.e., the double floating-point format). Furthermore, in this study, the GPU was simultaneously used as the display card in the experiments, which affected its performance. The use of an additional supercomputing GPU will be beneficial for deep learning. However, doing so would not have helped in this study because the small mainframe used in the study, the size of which was half that of a standard desktop, had only one PCI-E slot. These practical problems indicate that to accelerate deep learning by using a GPU, large, expensive equipment is required, which is impractical for executing deep learning on small devices such as the Internet of Things terminals or mobile devices.

Training loss The training data predicted by DeepTTE were significantly poor, indicating that DeepTTE performed poorly at the fundamental level. The training data already inputted in the training stage. Moreover, a deep learning model is usually trained with five-fold cross-validation, which was not conducted in our experiments because DeepTTE was already significantly poor at predicting the training data. 


\section{MORE APPLICATIONS}

This section presents two applications of hypercube clustering. The first application involves the identification of popular routes. The second application is used to illustrate the trend of hurricanes on the east coast of the U.S.

\subsection{Popular Routes}

The first application involves finding popular routes from trajectories, which could be used for travel recommendations, traffic control, or behavior analysis. This study employed User 3 (175 trajectories) in the Geo-life dataset in this demonstration. The date of each trajectory was removed, but the time was left. The parameters in this example were as follows: $\tau=3600$ seconds, $d_{x}=0.00117$ (100 meters) and $d_{y}=0.0009(100$

meters),$\delta=\frac{1}{6} \Phi$. This study set $p$ to 0.1 to obtain the top- $10 \%$ most popular sub-trajectories. 
In FIGURE 29, the green dots indicate the center position of each Hypercube, but not the original trajectory in cases where there were too many points to draw. FIGURE 30 presents a zoomed-in image from FIGURE 29. Each arrow represents a cluster of common sub-trajectories, which is the average of hypercubes in a cluster.

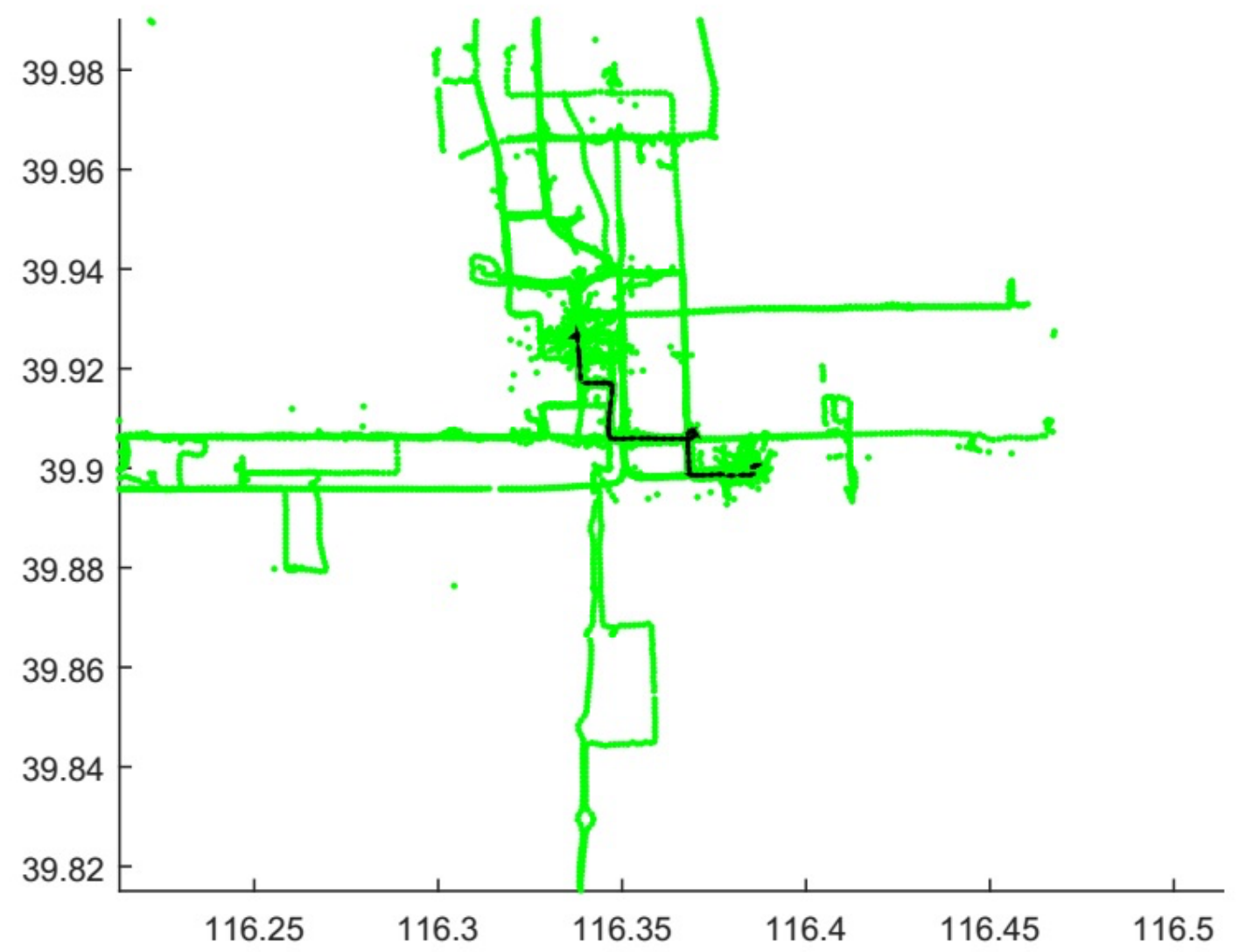

FIGURE 29. The result is presented on a two-dimensional plot. The black dashed line indicates the $10 \%$ most popular sub-trajectories. The green dots indicate the central position of each Hypercube. 


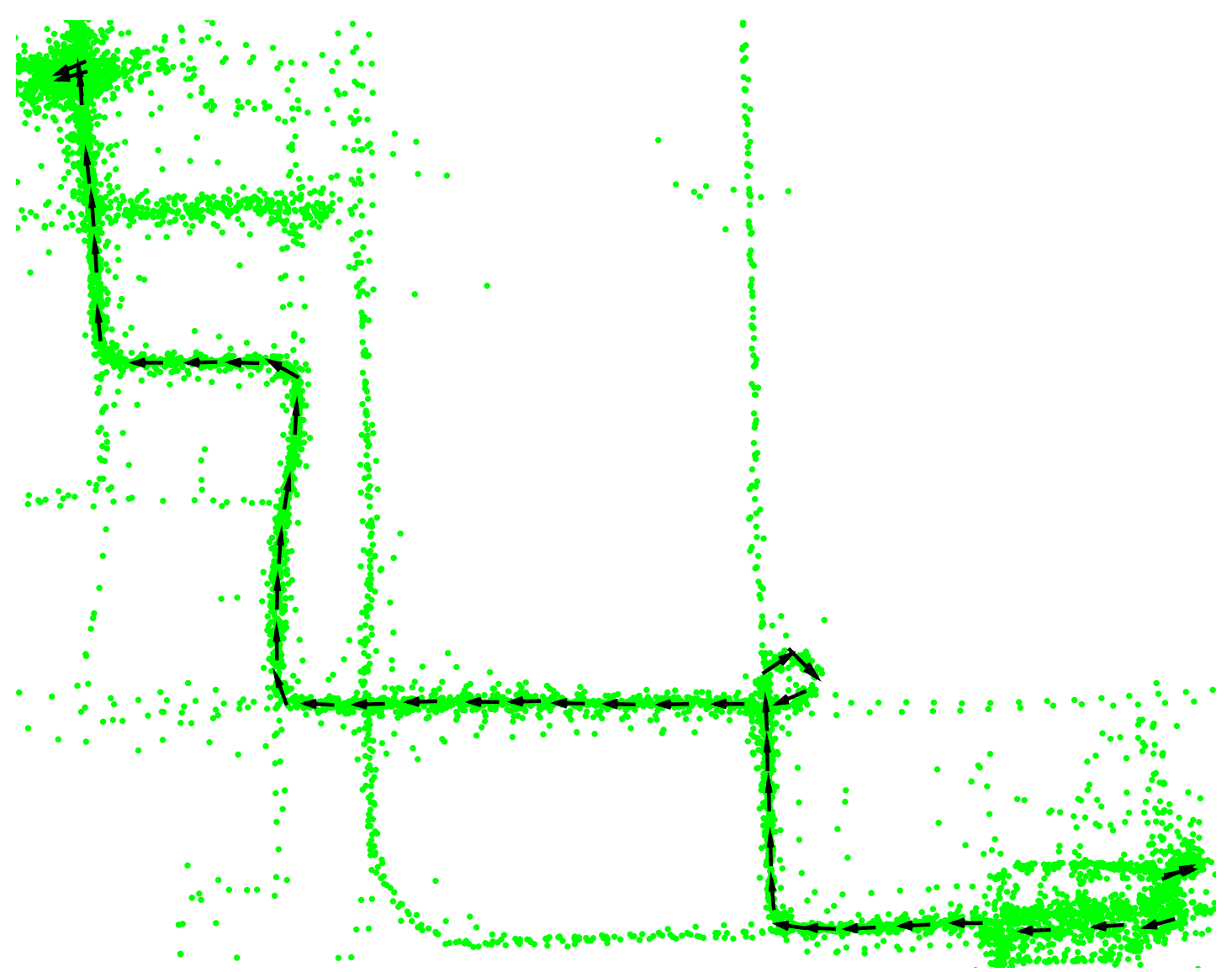

FIGURE 30. Zoomed-in view of results from the previous plot. The black dashed lines are formed by a series of arrows to show the route and direction. 
FIGURE 31 presents temporal dimensions on the Z-axis. The black arrows have many sub-trajectories (green points) surrounding them, indicating that targets passed through these roads frequently and within an hour of each other.

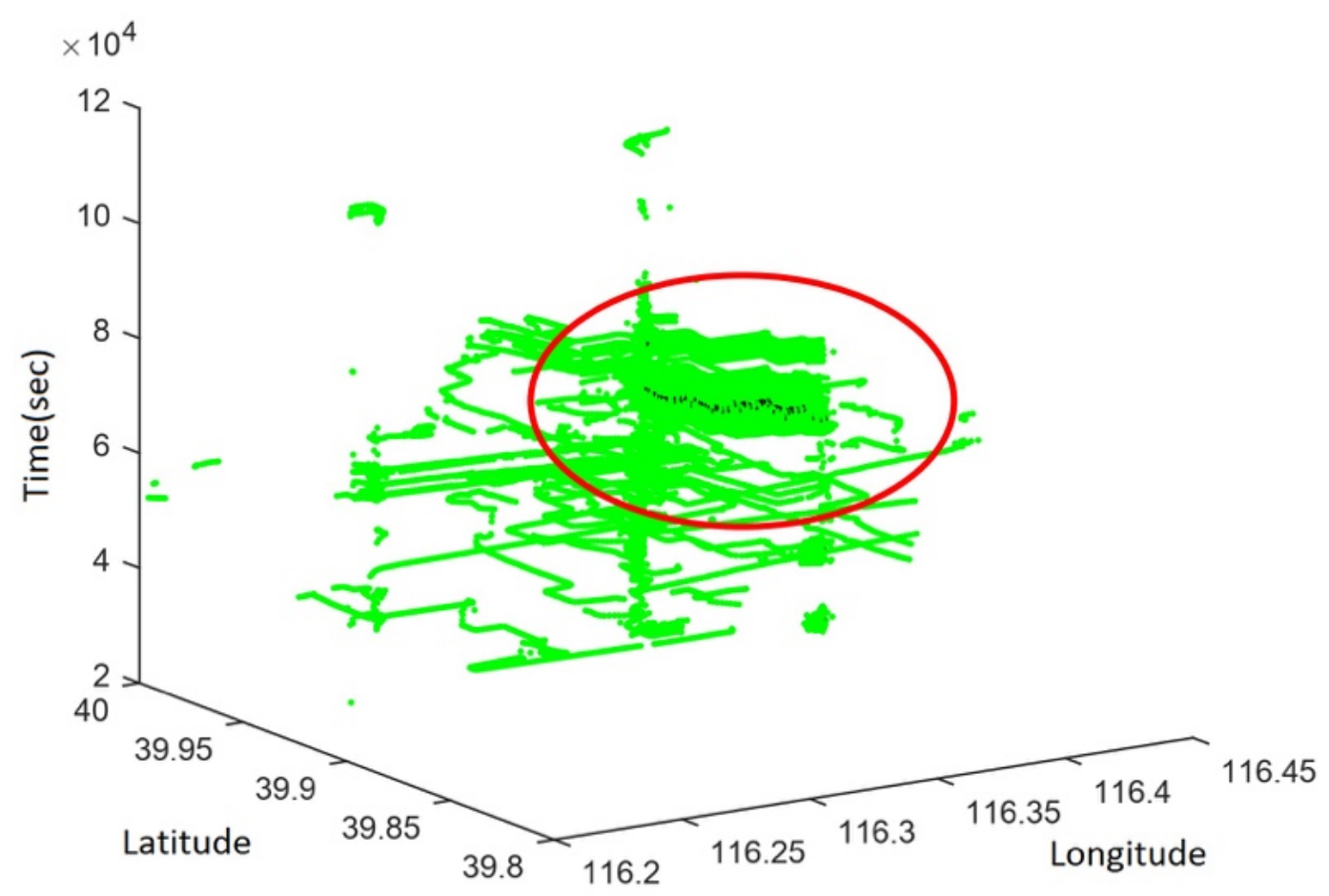

FIGURE 31. Popular sub-trajectories with the temporal distribution. The black dots indicate arrows, and the green points indicate the center of hypercubes, as in the previous figure. The third dimension (height) is temporal space, ranging from 20,000 seconds to 120,000 seconds(5:30 a.m. to next day 9:20 a.m.). Longitude ranged from 116.2 to 116.45 . Latitude ranged from 39.8 to 40. 
Increasing $p$ would result in a larger number of popular sub-trajectories. FIGURE 32 shows the top-50\% most popular sub-trajectories, where $p=0.5$. FIGURE 33 shows the top- $80 \%$ most popular sub-trajectory, where $p=0.8$. The popular subtrajectories that appear in FIGURE 33 exceed the scope of FIGURE 32.

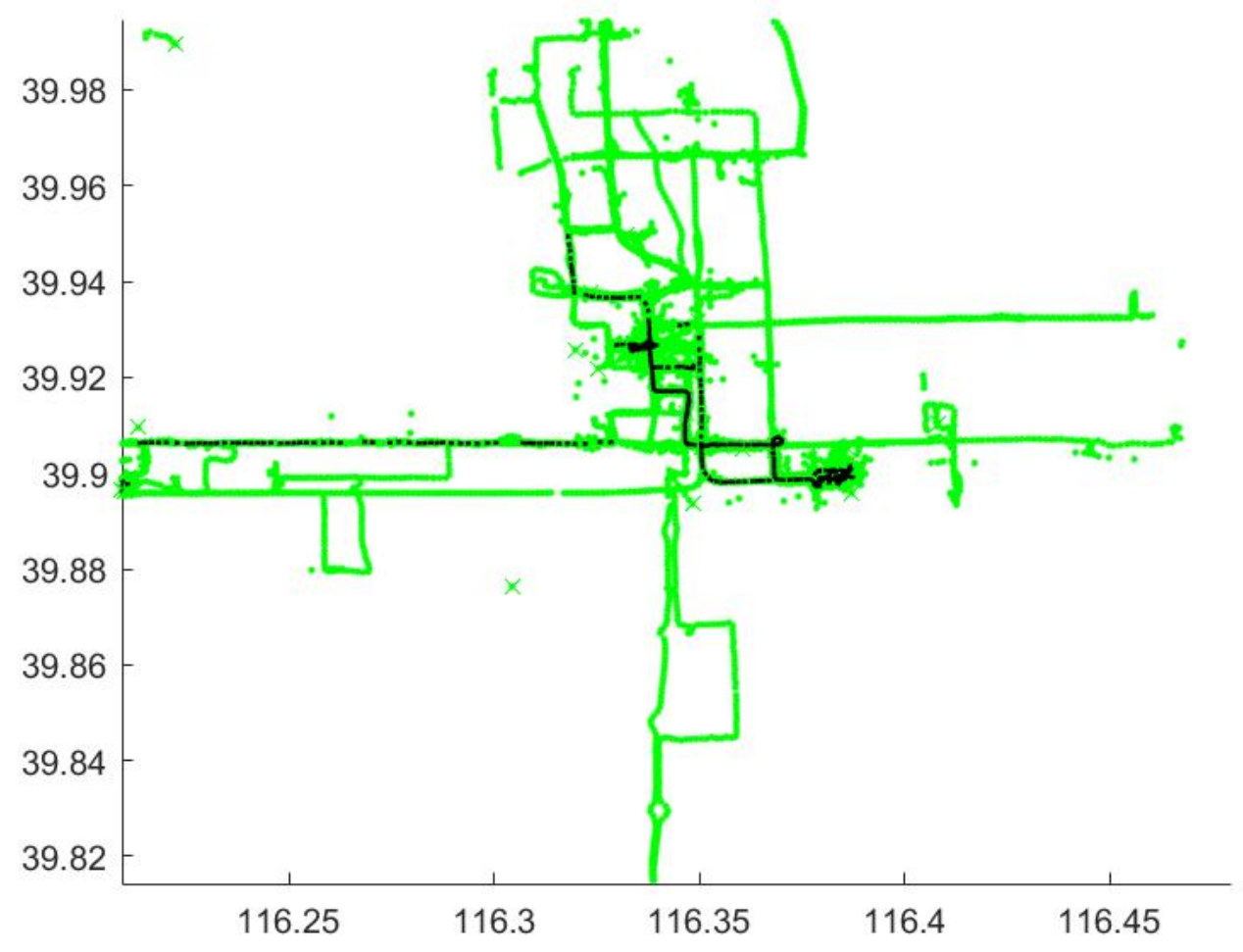

FIGURE 32. Results obtained with $\mathrm{p}=0.5$. The black dashed lines indicate $50 \%$ most popular sub-trajectories. The green dots indicate the central position of each Hypercube. 


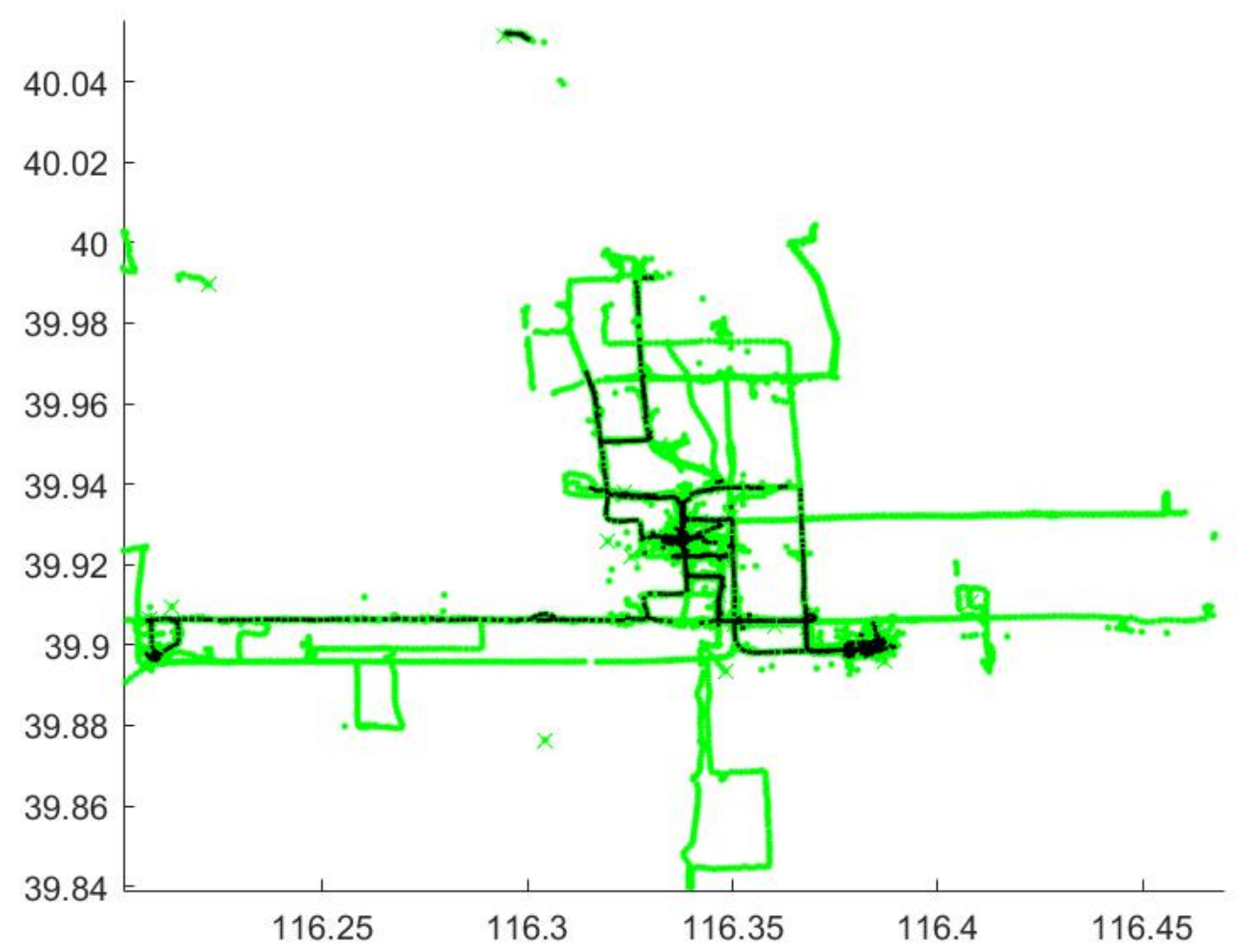

FIGURE 33. Results obtained with $p=0.8$. The black dashed lines indicate $80 \%$ most popular sub-trajectories. The green dots indicate the central position of each Hypercube. 


\subsection{The Hurricane Movement}

$\ulcorner$ The example below illustrates the use of Hypercube clustering to identify trends in the tracking of hurricanes. The data set used in this experiment was drawn from hurricane trajectories near the east coast of the U.S. between 1950 and $2004^{5}$. The trajectory points in FIGURE 34 are raw data related to hurricane trajectories between days 171 and 200 (i.e., from the middle of June to the middle of July in each year). The blue arrows indicate the trend of the hurricanes. Each arrow is a sub-trajectory cluster. The hurricane trajectory (cyan points) with " $\mathrm{X}$ " symbols act as endpoints. The background was obtained from GPSVisualizer.com based on Google terrain. The size of the arrows varies according to the number of members represented by that cluster. A larger arrow indicates a stronger tendency. $\lrcorner[4]$

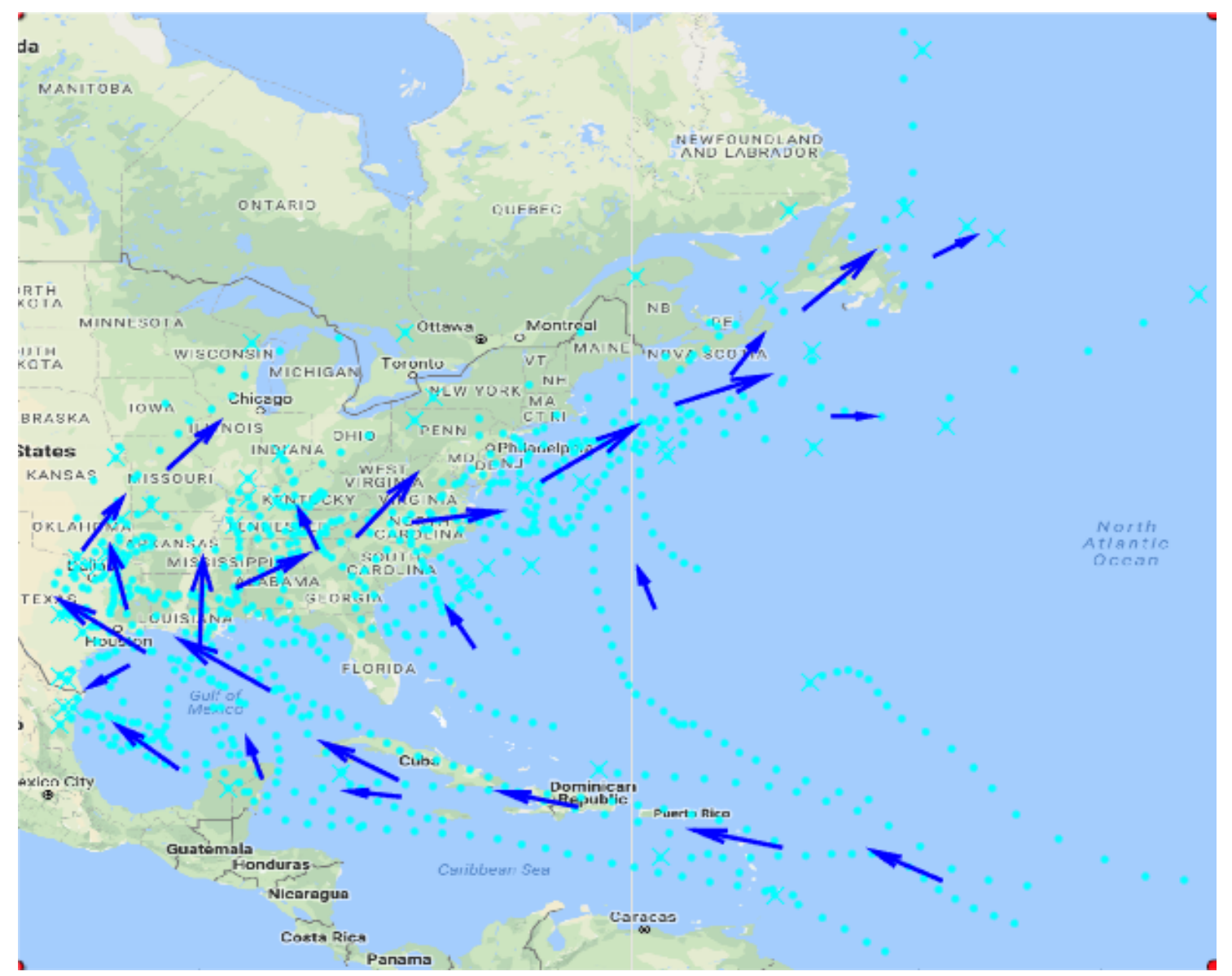

FIGURE 34. Hurricane trajectories and underlying trends.

\footnotetext{
${ }^{5} \mathrm{http} / / /$ weather.unisys.com/hurricane/atlantic
} 
This study selected hurricanes from day 171 to 200 because they provide many long trajectories, thereby ensuring that the results are definitive. This study selected the range by manually scanning the temporal distribution of trajectories from day 1 to 365 . This study defined a 30day time window, sliced the data set by moving the window every five days, and watched the data slice on a spatial graph to choose an appropriate time period. FIGURE 35 presents the distribution in a temporal space used in the selection process. As shown in the figure, hurricanes between days 150 and 350 presented a normal distribution in this range.

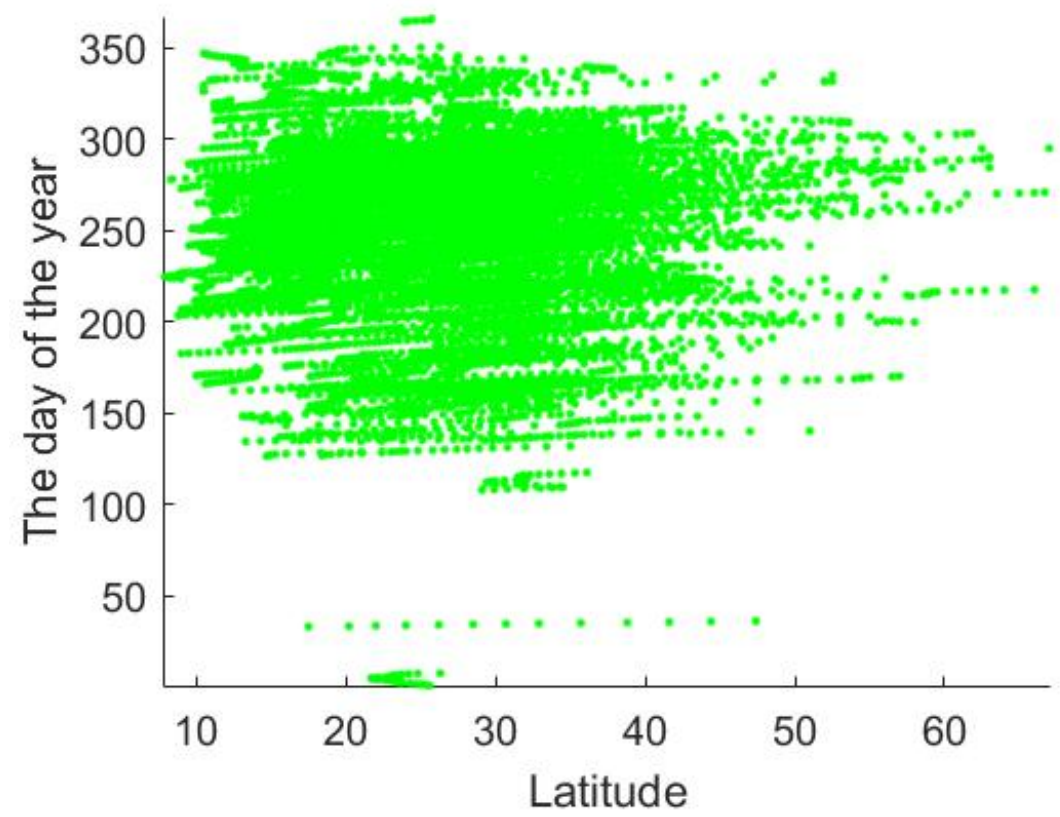

FIGURE 35. Temporal distribution of all hurricanes included in the raw data.

This study used hypercube clustering to generate a graph and compare it with the findings in [14] [15]. The graph obtained using hypercube clustering presents more information than that of the graphs in the previous studies. 


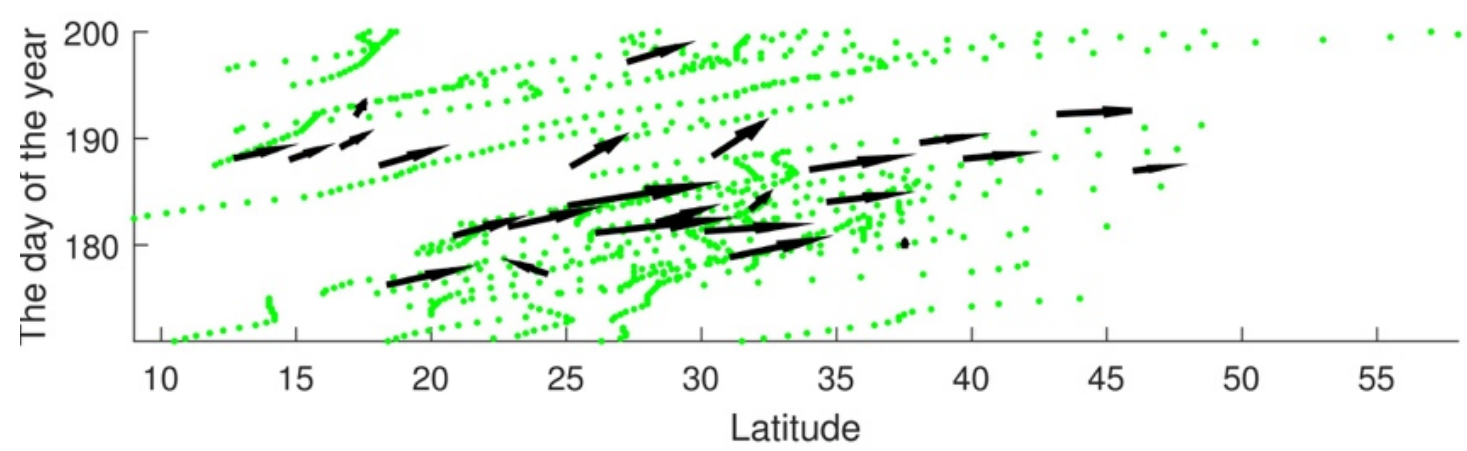

FIGURE 36. Temporal distribution of hurricanes between days 171 and 200. The dark arrows indicate the results obtained using hypercube clustering.

FIGURE 36 presents the clustering results in a temporal space, showing only time and latitude dimensions. The arrows proceed from low latitude to high, which is the same as the direction of the green trajectory points.

$\left\ulcorner\right.$ The parameters in this example were as follows: $d_{x}=5, d_{y}=5, \delta=30$ degree, and $\tau=30$ days. $p$ was set at 0.9999 . The longitude range in FIGURE 34 is 100; therefore, $\boldsymbol{d}$ was set to $5 . \tau$ can be set to cover the entire range of the temporal space of trajectories. In the example, the temporal range of the data is 30 days; therefore, $\tau=30$. $p$ can be set at 0.9999 to ensure the removal Hypercubes that do not share any intersections. However, it is strongly suggested that the user understands the meaning of the parameters in order to obtain the desired results. 
The value of $\varepsilon$ can be varied to obtain different results and show different graphs.

If $d_{x}$ and $d_{y}$ are 10 in this application, then the resolution will be low, as shown in FIGURE 37. If $d_{x}$ and $d_{y}$ are 2 , then the resolution will be high, as shown in FIGURE 38. $\lrcorner[4]$

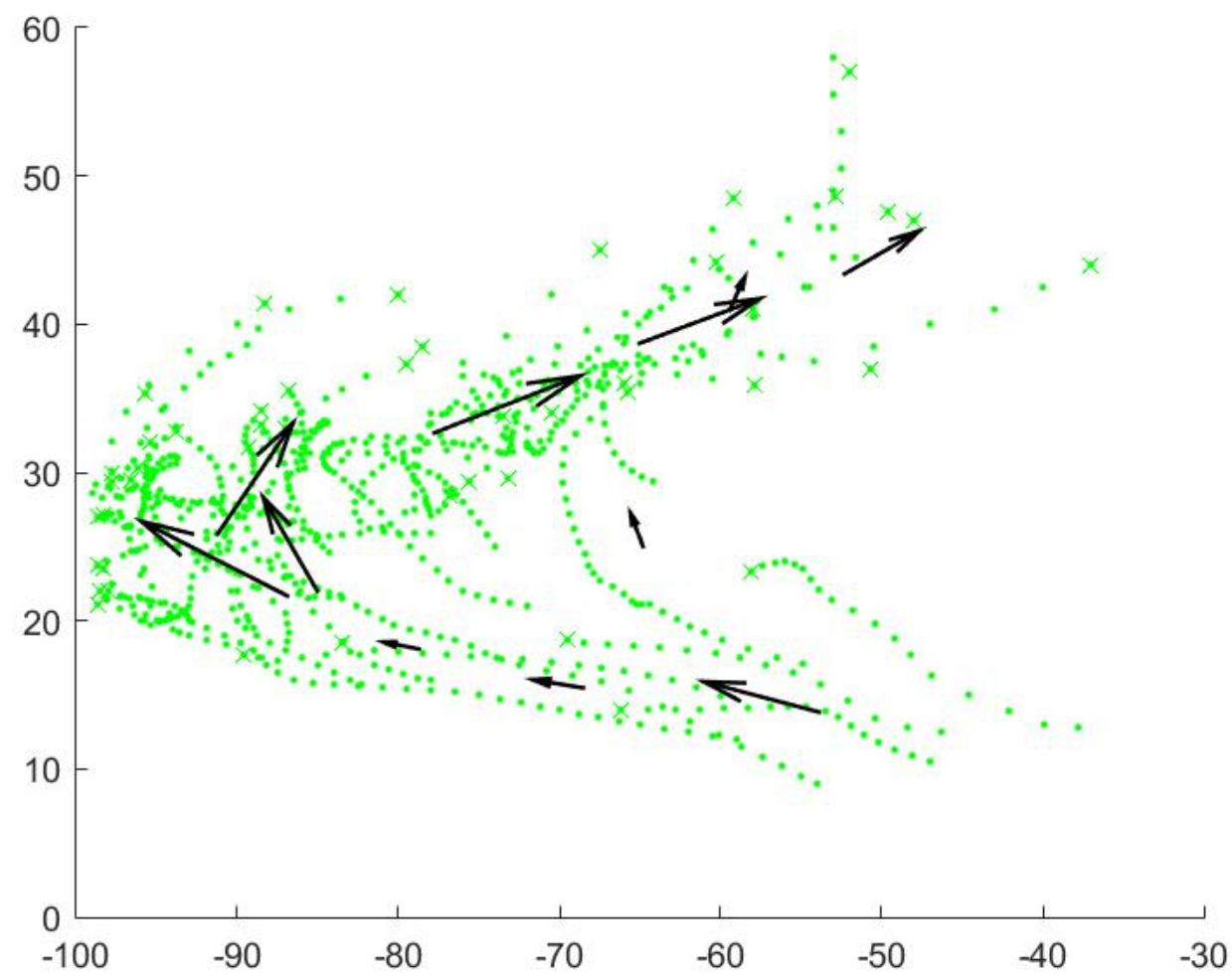

FIGURE 37. Trends in hurricane movement when $d_{x}=10$ and $d_{y}=10$. 


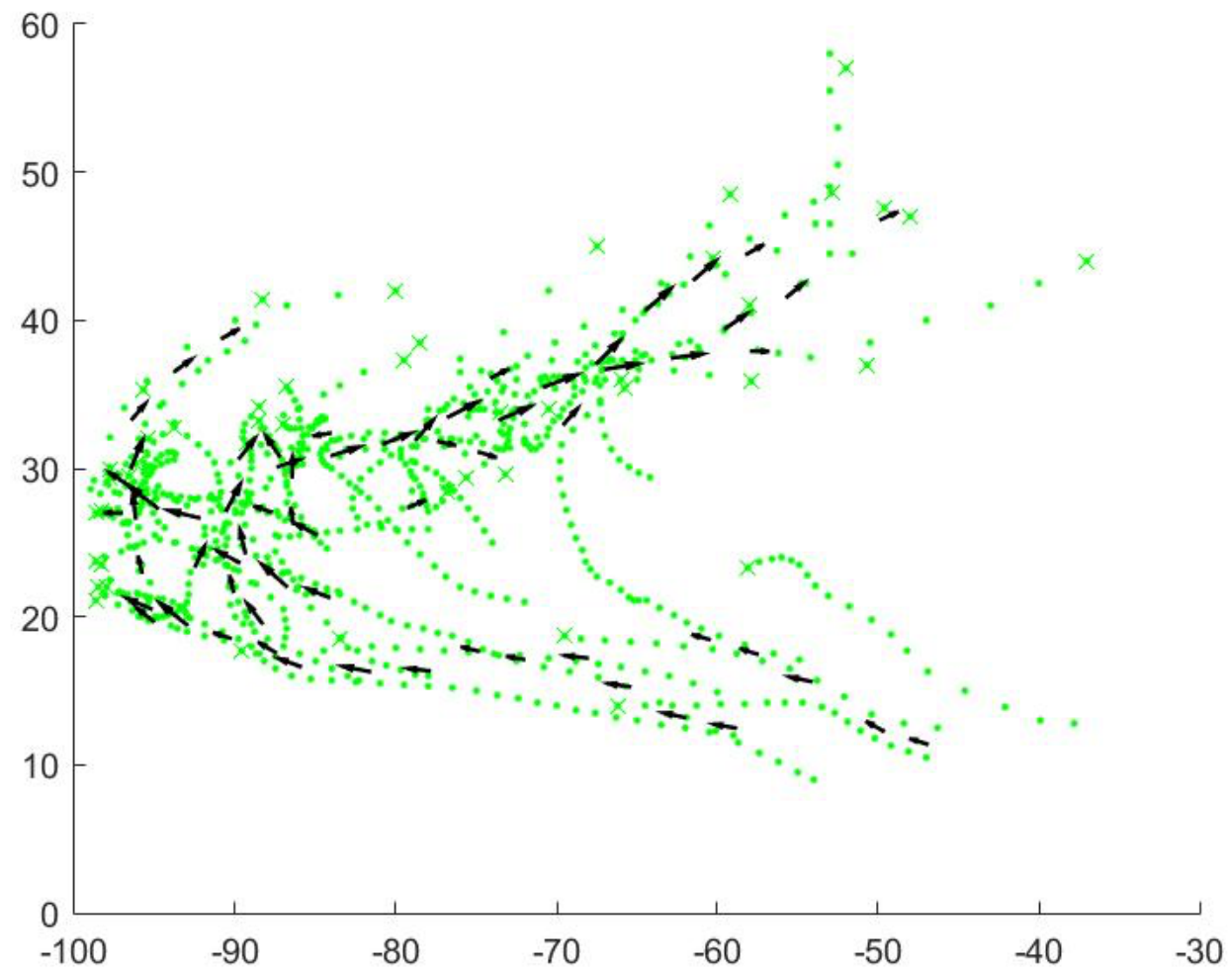

FIGURE 38. Trends in hurricane movement when $d_{x}=2$ and $d_{y}=2$. 


\section{CONCLUSION}

This study designs a novel approach, Hypercubes Clustering, to identifying common sub-trajectories. The proposed method is able to efficiently find common subtrajectories from multiple trajectories within a spatiotemporal space. Hypercubes Clustering is further applied to HyperETA to predict ETA for a given trajectory.

Experiment results demonstrate that this approach outperforms grid clustering in terms of identifying common sub-trajectories. This method also generates far more useful information than that of other schemes, including temporal factors and direction. HyperETA significantly outperformed DeepTTE and DeepTTE-GPU. The MATLAB source $\operatorname{code}^{6}$ and the $\mathrm{C}++$ source $\operatorname{code}^{7}$ of Hypercube Clustering have also been released on Github. The software of HyperETA ${ }^{8}$ and DeepTTE ${ }^{9}$ in the experiments have also been released.

${ }^{6}$ http://github.com/oscarhsu/TrajCubeClus

7 http://github.com/oscarhsu/TrajCubeClusCpp

8 https://github.com/oscarhsu/HyperETA

9 https://github.com/oscarhsu/DeepTTE 


\section{REFERENCES}

[1] J.-G. Lee, J. Han, and K.-Y. Whang, "Trajectory Clustering: A Partition-and-group Framework," in Proceedings of the 2007 ACM SIGMOD International Conference on Management of Data, New York, NY, USA, 2007: ACM, in SIGMOD '07, pp. 593604. [Online]. Available: http://doi.acm.org/10.1145/1247480.1247546. [Online]. Available: http://doi.acm.org/10.1145/1247480.1247546

[2] Y. Zheng, L. Capra, O. Wolfson, and H. Yang, "Urban computing: concepts, methodologies, and applications," ACM Transactions on Intelligent Systems and Technology (TIST), vol. 5, no. 3, p. 38, 2014.

[3] W. He, K. Hwang, and D. Li, "Intelligent Carpool Routing for Urban Ridesharing by Mining GPS Trajectories," Intelligent Transportation Systems, IEEE Transactions on, vol. 15, no. 5, pp. 2286-2296, 2014.

[4] O. L. Hsu and C.-R. Lee, "Common Sub-Trajectory Clustering via Hypercubes in Spatiotemporal Space," IEEE Access, vol. 8, pp. 23369-23377, 2020, doi: 10.1109/ACCESS.2020.2968150.

[5] O. L. J. Hsu and C.-R. Lee, "Approach for Finding Ridesharing Paths in Spatiotemporal Space," in SMART 2016: The Fifth International Conference on Smart Systems, Devices and Technologies(include URBAN COMPUTING 2016), L. Berntzen, Ed., 2016: IARIA, pp. 37-43.

[6] M. d. Berg, O. Cheong, M. v. Kreveld, and M. Overmars, Computational Geometry: Algorithms and Applications. Springer-Verlag TELOS, 2008, p. 386.

[7] D. Wang, J. B. Zhang, W. Cao, J. Li, Y. Zheng, and Aaai, "When Will You Arrive? Estimating Travel Time Based on Deep Neural Networks," in AAAl-18, 2018, pp. 2500-2507. [Online]. Available: <Go to ISI>://WOS:000485488902071. [Online]. Available: $<$ Go to $|S|>: / / W O S: 000485488902071$

[8] L. Tran, M. Y. Mun, M. Lim, J. Yamato, N. Huh, and C. Shahabi, "DeepTRANS: a deep learning system for public bus travel time estimation using traffic forecasting," Proc. VLDB Endow., vol. 13, no. 12, pp. 2957-2960, 2020, doi: $10.14778 / 3415478.3415518$.

[9] J. Hu, B. Yang, C. Guo, C. S. Jensen, and H. Xiong, "Stochastic Origin-Destination Matrix Forecasting Using Dual-Stage Graph Convolutional, Recurrent Neural Networks," in 2020 IEEE 36th International Conference on Data Engineering (ICDE), 20-24 April 2020 2020, pp. 1417-1428, doi: 10.1109/ICDE48307.2020.00126.

[10] J. Qiu, L. Du, D. Zhang, S. Su, and Z. Tian, "Nei-TTE: intelligent traffic time estimation based on fine-grained time derivation of road segments for smart city," IEEE Transactions on Industrial Informatics, vol. 16, no. 4, pp. 2659-2666, 
2019.

[11] R. Dai, S. Xu, Q. Gu, C. Ji, and K. Liu, "Hybrid Spatio-Temporal Graph Convolutional Network: Improving Traffic Prediction with Navigation Data," in Proceedings of the 26th ACM SIGKDD International Conference on Knowledge Discovery \& Data Mining, 2020, pp. 3074-3082.

[12] K. Fu, F. Meng, J. Ye, and Z. Wang, "Compacteta: A fast inference system for travel time prediction," in Proceedings of the 26th ACM SIGKDD International Conference on Knowledge Discovery \& Data Mining, 2020, pp. 3337-3345.

[13] Q. Chen et al., "A Survey on an Emerging Area: Deep Learning for Smart City Data," IEEE Transactions on Emerging Topics in Computational Intelligence, vol. 3, no. 5, pp. 392-410, 2019, doi: 10.1109/TETCI.2019.2907718.

[14] C.-H. Chen, C.-R. Lee, and W. C.-H. Lu, "Smart in-car camera system using mobile cloud computing framework for deep learning," Vehicular Communications, vol. 10, pp. 84-90, 2017.

[15] C.-H. Chen, C.-R. Lee, and W. C.-H. Lu, "A mobile cloud framework for deep learning and its application to smart car camera," in International conference on internet of vehicles, 2016: Springer, pp. 14-25.

[16] K. Buchin, M. Buchin, M. Van Kreveld, and J. Luo, "Finding long and similar parts of trajectories," Computational Geometry, vol. 44, no. 9, pp. 465-476, 2011.

[17] M. Vlachos, G. Kollios, and D. Gunopulos, "Discovering similar multidimensional trajectories," in Data Engineering, 2002. Proceedings. 18th International Conference on, 2002, pp. 673-684.

[18] L. Chen, z. ।"O, M. Tamer, and V. Oria, "Robust and fast similarity search for moving object trajectories," in Proceedings of the 2005 ACM SIGMOD international conference on Management of data, 2005, pp. 491-502.

[19] S. Nutanong, E. H. Jacox, and H. Samet, "An incremental Hausdorff distance calculation algorithm," Proceedings of the VLDB Endowment, vol. 4, no. 8, pp. 506-517, 2011.

[20] E. Keogh and C. A. Ratanamahatana, "Exact indexing of dynamic time warping," Knowledge and information systems, vol. 7, no. 3, pp. 358-386, 2005.

[21] G. A. ten Holt, M. J. T. Reinders, and E. A. Hendriks, "Multi-Dimensional Dynamic Time Warping for Gesture Recognition," ed, 2007.

[22] N. Ferreira, J. T. Klosowski, C. E. Scheidegger, and C. a. Silva, udio T., "Vector Field k-Means: Clustering Trajectories by Fitting Multiple Vector Fields," in Computer Graphics Forum, 2013, vol. 32, pp. 201-210.

[23] R. Cheng et al., "Managing uncertainty in spatial and spatio-temporal data," in Data Engineering (ICDE), 2014 IEEE 30th International Conference on, 2014, pp. 
1302-1305.

[24] D. Huang, C.-D. Wang, J. Wu, J.-H. Lai, and C. K. Kwoh, "Ultra-Scalable Spectral Clustering and Ensemble Clustering," IEEE Transactions on Knowledge and Data Engineering, 2019.

[25] G. Atluri, A. Karpatne, and V. Kumar, "Spatio-Temporal Data Mining: A Survey of Problems and Methods," ACM Comput. Surv., vol. 51, no. 4, pp. 83:1-83:41, August 2018. [Online]. Available: http://doi.acm.org/10.1145/3161602.

[26] J. Bian, D. Tian, Y. Tang, and D. Tao, "A survey on trajectory clustering analysis," ed, 2018.

[27] P. Tampakis, N. Pelekis, C. Doulkeridis, and Y. Theodoridis, "Scalable Distributed Subtrajectory Clustering," CoRR, vol. abs/1906.06956, 2019. [Online]. Available: http://arxiv.org/abs/1906.06956.

[28] M. R. Evans, D. Oliver, S. Shekhar, and F. Harvey, "Fast and exact network trajectory similarity computation: a case-study on bicycle corridor planning," in Proceedings of the 2nd ACM SIGKDD International Workshop on Urban Computing, 2013, p. 9.

[29] M. R. Evans, D. Oliver, S. Shekhar, and F. Harvey, "Summarizing trajectories into k-primary corridors: a summary of results," in Proceedings of the 20th International Conference on Advances in Geographic Information Systems, 2012, pp. 454-457.

[30] S. Ma, Y. Zheng, and O. Wolfson, "Real-time city-scale taxi ridesharing," Knowledge and Data Engineering, IEEE Transactions on, vol. 27, no. 7, pp. 17821795, 2015.

[31] B. Han, L. Liu, and E. R. Omiecinski, "A Systematic Approach to Clustering Whole Trajectories of Mobile Objects in Road Networks," IEEE Transactions on Knowledge and Data Engineering, 2017.

[32] M. Furuhata, M. Dessouky, n. Ord\'o, ez, Fernando, M.-E. Brunet, X. Wang, and S. Koenig, "Ridesharing: The state-of-the-art and future directions," Transportation Research Part B: Methodological, vol. 57, pp. 28-46, 2013.

[33] D. S. Hochbaum and P. Baumann, "Sparse computation for large-scale data mining," in Big Data (Big Data), 2014 IEEE International Conference on, 2014, pp. 354-363.

[34] Y. Ong and A. Gupta, "AIR5: Five Pillars of Artificial Intelligence Research," IEEE Transactions on Emerging Topics in Computational Intelligence, vol. 3, no. 5, pp. 411-415, 2019, doi: 10.1109/TETCI.2019.2928344.

[35] M. Veres and M. Moussa, "Deep Learning for Intelligent Transportation Systems: A Survey of Emerging Trends," IEEE Transactions on Intelligent Transportation 
Systems, vol. 21, no. 8, pp. 3152-3168, 2020, doi: 10.1109/TITS.2019.2929020.

[36] P. Molchanov, X. Yang, S. Gupta, K. Kim, S. Tyree, and J. Kautz, "Online Detection and Classification of Dynamic Hand Gestures with Recurrent 3D Convolutional Neural Networks," in 2016 IEEE Conference on Computer Vision and Pattern Recognition (CVPR), 27-30 June 2016 2016, pp. 4207-4215, doi: 10.1109/CVPR.2016.456.

[37] L. H. Gilpin, D. Bau, B. Z. Yuan, A. Bajwa, M. Specter, and L. Kagal, "Explaining Explanations: An Overview of Interpretability of Machine Learning," in 2018 IEEE 5th International Conference on Data Science and Advanced Analytics (DSAA), 13 Oct. 2018 2018, pp. 80-89, doi: 10.1109/DSAA.2018.00018.

[38] Z. Zeng, C. Miao, C. Leung, and C. J. Jih, "Building more explainable artificial intelligence with argumentation," in Proc. 23rd AAAI/SIGAI Doctoral Consortium, 2018, pp. 8044-8045.

[39] C. M. Bishop, "Mixture Models and EM," in Pattern recognition and machine learning: springer, 2006, ch. 9.

[40] M. Ester, H.-P. Kriegel, J. Sander, and X. Xu, "A density-based algorithm for discovering clusters in large spatial databases with noise," in Kdd, 1996, vol. 96, no. 34 , pp. $226-231$.

[41] Dynamic Time Warping Python Module. (2019). GitHub. Accessed: Dec 11. [Online]. Available: https://github.com/pierre-rouanet/dtw

[42] S. Narang et al., "Mixed precision training," in Proc. 6th Int. Conf. on Learning Representations (ICLR), 2018. 